\title{
Die Entwicklungsgeschichte des Augapfels von Megalobatrachus japonicus mit besonderer Berücksichtigung auf die Entstehung des Skleralknorpels sowie der Kornealgefässe.
}

\author{
Von \\ Kakichi Sawada. \\ Aus dem Anatomischen Institut der. Keio Universität, Tokyo. \\ Mit 37 Textfiguren.
}

Wenmgleich das Sehorgan des Megalobatrachus japonicus seit langem von vielen Autoren, wie Schmidt-Goddart-van der Hoeven, Rejsek, Lauber, Murata, Ito, Tsuzaki'und Yano u.a. vielfach untersucht worden ist, bleibt doch dessen Entwicklungsgeschichte ganz unberücksichtigt.

So habe ich mich in der vorliegenden Untersuchung mit der Entstehung des Augapfels und besonders des ersten Auftretens des Skleralknorpels sowie mit der frühzeitigen Vaskularisierung der Kornea des betreffenden Tieres beschäftigt.

\section{Material und Methode.}

Das Material stammt aus Okajima-Tago-Serien. Die Larven in 10 Stadien von 8,5 bis $179 \mathrm{~mm}$ Körperlänge waren grösstenteils mit Formalin und zum Teil mit Bouins Flüssigkeit fixiert; sie wurden alle in Paraffin eingebettet und zum grössten Teil. in senkrechte, nur einige in horizontale Serien von $20 \mu$ Dicke geschnitten.

Dic $30 \mathrm{~mm}, 80 \mathrm{~mm}$ und $179 \mathrm{~mm}$ langen Exemplare wurden, nach 
der Entkalkung mit 3\%iger Salpetersäure, in Zclloidin eingebettet und in senkrechte Schnitte von $30 \mu$ bis $50 \mu$ Dicke zerlegt.

Die Schnitte wurden mit Eisenhämatoxylin nach Wreigert und Hämatoxylin-Eosin gefärbt. Ich habe die folgenden 10 Wachsmodelle des Augapfels von 10 Stadion in 50 oder 100, facher Vergrösserung rekonstruiert. Für das Anbringen der Richtzeichen wurde der Beschneiderritzer von Okajima angewandt.

Dic Übersicht der rekonstruierten Modelle.

\begin{tabular}{|c|c|c|c|c|c|c|c|c|c|c|}
\hline Stadien & 1 & 2 & 3 & 4 & 5 & 6 & 7 & 8 & 9 & 10 \\
\hline Körperlänge & $8,5 \mathrm{~mm}$ & $11 \mathrm{~mm}$ & $19 \mathrm{~mm}$ & $20 \mathrm{~mm}$ & $20 . \overline{\mathrm{mm}}$ & $23.5 \mathrm{~mm}$ & $26.5 \mathrm{~mm}$ & $30 \mathrm{~mm}$ & $80 \mathrm{~mm}$ & $179 \mathrm{~mm}$ \\
\hline Seite & Links & .. & ., &. & ,. & ," & .. & $\begin{array}{l}\text { Beider- } \\
\text { seits }\end{array}$ & Rechts & , \\
\hline Schnittdicke & $20 \mu$ & , & , &.. & ,. & , & .. & $30 \mu$ & , & $50 \mathrm{us}$ \\
\hline Vergrösserung & 50 fach & ," & , & , & .. & .. & .. & $66 \mathrm{fach}$ & ., & 40 fach \\
\hline
\end{tabular}

\section{Stadienbeschreibung.}

1) Starlium $1 \quad(8,5 \mathrm{~mm}$ lange Larve).

Das Riechfeld ist kaum erkemnbar und das Hörbläschen fast areschlosson.

Die primäre Augenblase (Fig. 1, $2,3)$ ist schon gut ausurebildet. Die ganze Gestalt des Bläschens ist beinahe oval und steht mit der Längsachse in sagittaler Richtung. Seine ventrale Oberfläche ist leicht konkav. Das Bläschen sclbst verbindet sich auf dicker Basis mit der Hirnblase. Er enthält eine relativ grosse Bläschenhöhle, die durch die weite Augrenstielhöhle mit dem Hirnventrikel kommuniziert. Die Dicke der ventralen und dorsalen sowie aller anderen Bläschenwände sind beinahe glcich (Fig. 4).

Die Aussenfläche der ventralen Wand ist konkay und dementspre-

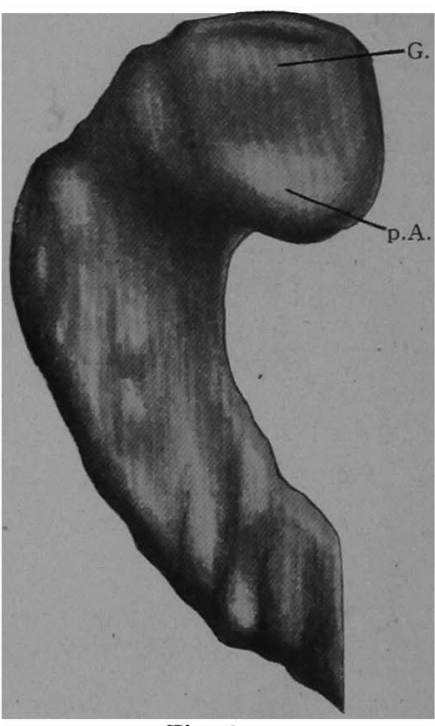

Fig. 1.

Iaterale Ansicht des Modells der rechten primären Augen. b)ase. Starlium 1, 50 mal rergrösisert modelliert und auf 25 mal verkleinert reproduziert. (i. (ichirn. p.A. Primäre Augenblase. 
chend konvex in der Blasenhöhle; auf der Dorsalwand sind diese Verhältnisse ganz umgekehrt. Die Stielhöhlo ist am weitesten im Grenzgebiet dersclben und des Hirnventrikels. Dor Sohventrikel vorschmälert sich allmählich nach d'm Distalende und hört zuletzt mit stumpfem Ende auf. Die beiderscitimen Augenbläschen sind ungefähr gleichgross und gleichförmig.

Die Masse des Augenbläschens sind wie folgt:

Der dorsoventrale Durchmesser (a. $0,48 \mathrm{~mm}(24 \mathrm{~mm})$,

Der nasotemporale Durchmesser

Der anteroposteriore Durchmesser

(a. $0,68 \mathrm{~mm}(34 \mathrm{~mm})$, (a) $0,44 \mathrm{~mm}(22 \mathrm{~mm})$.

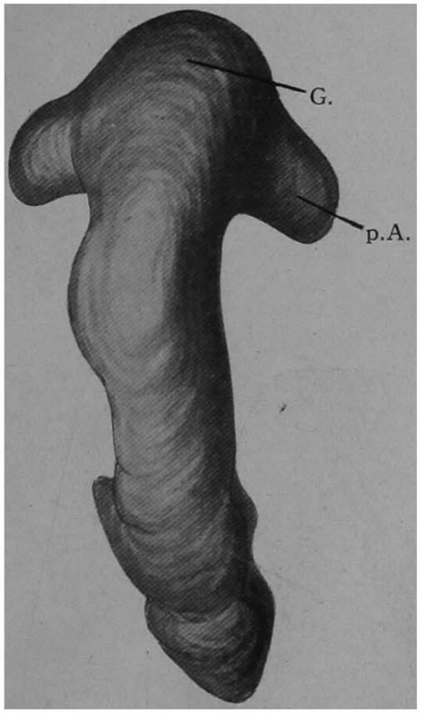

Fig. 2.

Vordere Insicht desselben Modells.

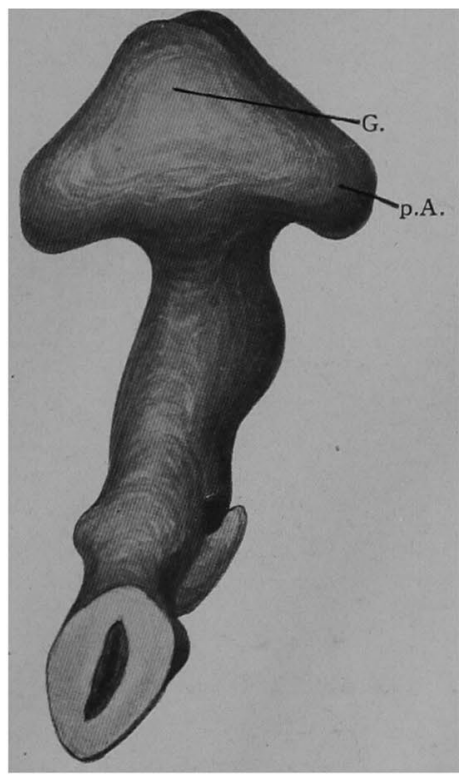

Fig. 3.

Hintere Ansicht desselben Modells.

Mikroskopisch : Das Ektoderm verbindet sich dicht mit der Blasenwand und zwischen den beiden sieht man keine Lücke. Die Blasenwand besteht aus mehrschichtigen Zylinderzellen und ihre Höhe nimmt allmählich gegen ihr distales Ende zu. Diese Zellen enthalten eine grosse Menge von Dotterkügelchen und Kernteilungsfiguren. In der Blasenwand sieht man auch spärliche Pigmentkörner. Das Ektoderm besteht aus zwei Schichten von Fpithelzellen und die Grundschicht zeigt noch keine V'erdickung.

2) Stadium 2 (11 $\mathrm{mm}$ lange Larve). 


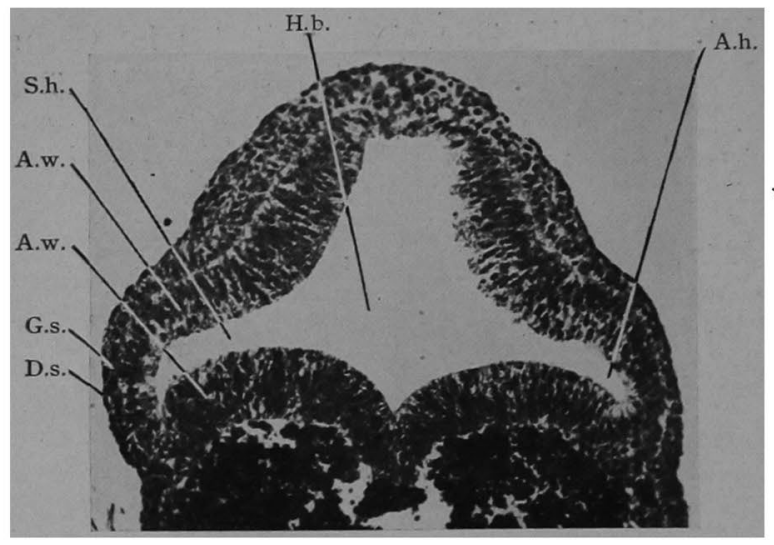

Fì. 4.

Frontaler Durchschnitt der primären Augenblates. (Stadium 1, V(ror. ca. 5i) mal).

HI.h. Hirnblase. S.h. sticlhöhle. A.w. Augenblasenwand. (i.s. (irundschicht. D.s. I)eckirhicht. A.h. Augenhöhle.

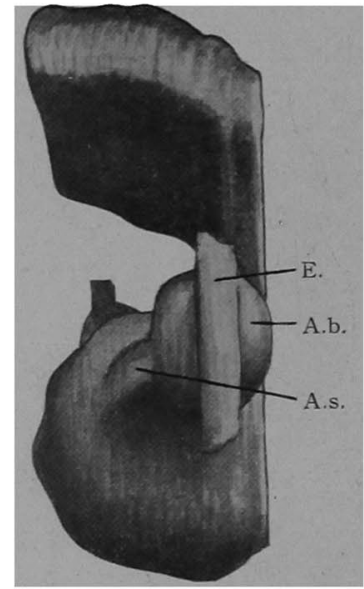

Fì. 5.

Iaterale Insicht des TIodells der rechten primären Augenblase. Stadium 2,5$)$ mal rerwrïsert modellicrt und auf 25 mal verkleinert reproduziert. A.s. Augensticl. E. Ektoderm. 1.h. Augenbecher.
Das Riechfeld ist schon gut ausgebildet und besteht aus mehreren Zellschichten. I)as Hörbläschen zeigt auf Srhnitten schon einen kleinen ovalen Binnemraum.

Das Augenbläschen (Fig. 5, $(;, 7)$ hat sich zum Augenbecher umgewandelt, der noch ganz seicht und an der A ussenwand etwas nach innen eingezogen ist. Dic Tiefe des Becher's beträgt etwa $0,05 \mathrm{~mm}$.

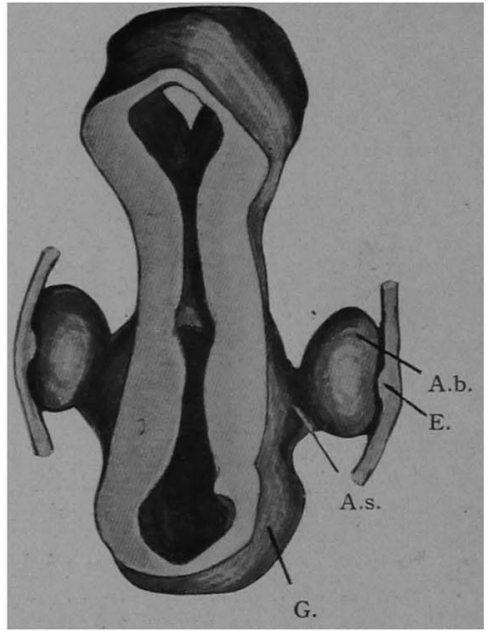

Fig. 6.

Vordere Insicht descolben Nodells.

Die Aussenwand des Becher's ist äussorst dick, während die Innenwand ganz dümn ist. Der Augenstiel ist in Grenzgebiet zum (rehirm 
abgeschnürt. Die Stielhöhle ist zum Sticlkanal verschmällert.

Die Nasse des Augenbechers sinu :

Der dorsoventralc Durchmesser

Der nasotemporale Durchrresser

Der anteroposteriore Durchmoss's.

Die Dicke des Augenstiels

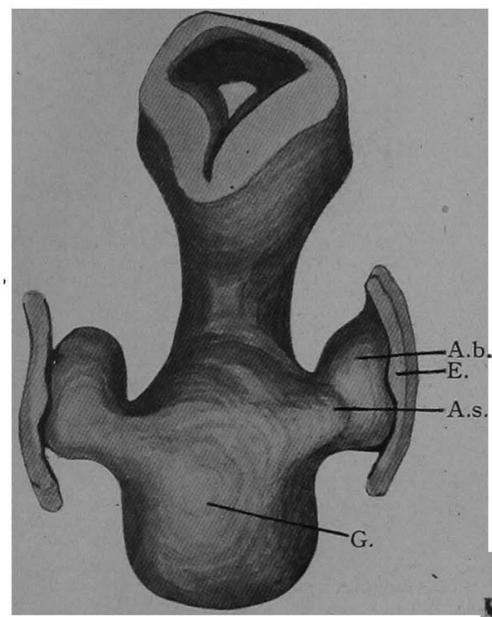

Fig. 7.

Hintere Ansicht desselben Norlells.

steht dagegen meistenteils aus einschichtigen kubischen Zellen. Alle diese Zellen enthalten reichliche Dotterkügelchen. ZWwischen diesen beiden Wänden befindet sich ein ganz dem Becher selbst entsprechend geformter Hohlraum.

Die grösste Entfernung beider Wände beläuft sich auf ca. 0,01 $\mathrm{mm}$. Die Linsenplatte besteht aus 2-3 Zellenlagen und die Deckschicht aus ein-

$$
\begin{aligned}
& \text { a. } 0,54 \mathrm{~mm}(27 \mathrm{~mm}) \text {, } \\
& \text { ca. 0,44 } \mathrm{mm}(22 \mathrm{~mm}) \text {, } \\
& \text { ca. 0,30 } \mathrm{mm}(15 \mathrm{~mm}) \text {, } \\
& \text { ca. } 0,28 \mathrm{~mm}(14 \mathrm{~mm}) \text {. }
\end{aligned}
$$

Mikroskopisch: Die Grundschicht des Ektoderms verdickt sich nach innen gegen den Becherraum (Linsenplatte) (Fig. 8). Zwischen der Aussenwand des Augenbechers und der Innenfläche der verdickten Grundschicht sieht man eine schmale Lücke.

Die Aussenwand des Bechers besteht aus mehreren Zellenschichten und wölbt sich gegen den Sehventrikel vor. Die Innenwand be-

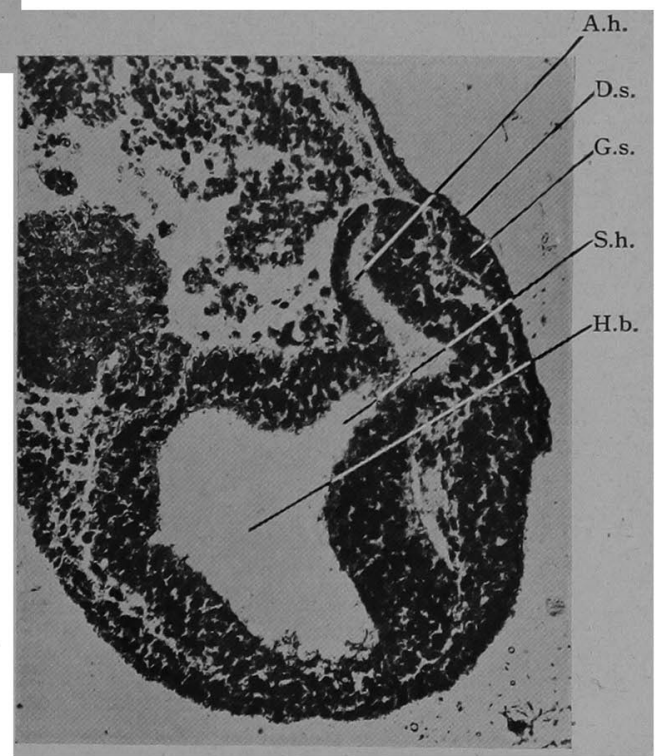

Fig. 8.

Frontaler Durchschnitt des rechten Augenbechers. (Stadium 2, Vergr. ca. 45 mal). H.b. Hirnblase. S.h. Sticlhöhle. A.h. Angenhöhle. (i.s. Grundschicht. D.s Deckschicht. 
schichtigen Plattenzellen. Die Deckschicht beteiligt sich durchaus nicht an der Linsenbildung und zieht nur die obere Fläche der Linsenplatte hinüber. Diese Zellen enthalten alle auch reichliche Dotterkügelchen Die Linsengrube ist noch gar nicht erkennbar. Die Aussenfläche des Linsenplatte ist ein wenig mehr hẻrorgewölbt al` daś ungrebende Haut. niveau.

3) Stadium 3 (19 $\mathrm{mm}$ lange Larre).

Die Riechgrube ist etwas tiefer geworden als vorher. Der Bimnen raum dess Hörblä-chens ist äusserst erweitert.

Die Linsenblass ist schon völlig ausgebildet (Fig. 9, 10).

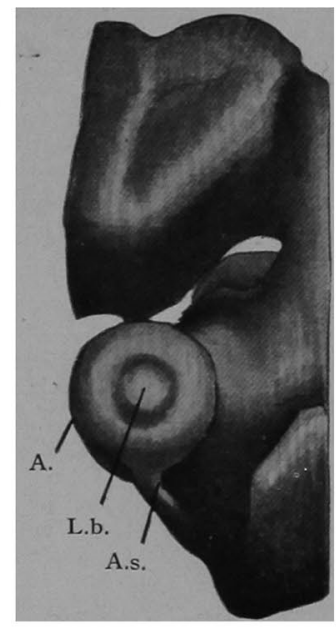

Fig. !

Laterale Ansicht des MIodells des rechten Auges. Stadium 3, 50 mal vergrösserte und auf 25 mal verkleinert reproduziert.

A. Auge. L.b. Linsenblase. A.s. Angenstiel.

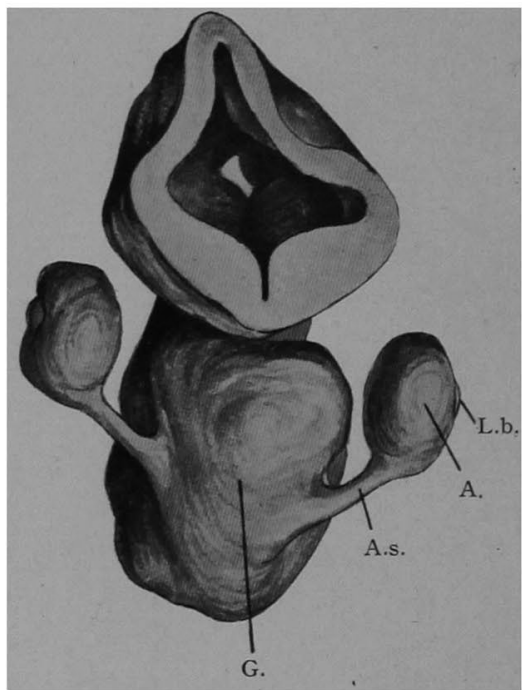

Fig. 10.

Hintere Ansicht desselben Modells.

G. (iehirn. A. Auge.

L.b. Linsenblase.

A.s. Augenstiel.

Die Masse der beiden Augen sind:

I. Das linke Auge.

Der dorsorentrale Durchmesere

Der nasotemporale Durchmesser

Der anteroposteriore Durchmesser

Die Länge des Augenstiel.

Die Dicke desscelben

ca. $0, \tilde{5} 4 \mathrm{~mm}(2 \overline{\mathrm{mm}})$,

ca. $0,50 \mathrm{~mm}(25 \mathrm{~mm})$,

ca. $0,34 \mathrm{~mm}(17 \mathrm{~mm})$,

ca. $0,28 \mathrm{~mm}(14 \mathrm{~mm})$,

ca. $0,06 \mathrm{~mm}$ ( $3 \mathrm{~mm})$. 
II. Das rechte Auge.

Der dorsoventrale Durchmesser ca. $0,52 \mathrm{~mm}(26 \mathrm{~mm})$,

Der nasotemporale Durchmesser ca. 0,48 $\mathrm{mm}(24 \mathrm{~mm})$,

Der anteroposteriore Durchmesser ca. $0,36 \mathrm{~mm}(18 \mathrm{~mm})$,

Die Lünge des Augenstiels ca. $0,28 \mathrm{~mm}(14 \mathrm{~mm})$,

Die Dicke desselben ca. $0,07 \mathrm{~mm}(3,5 \mathrm{~mm})$.

Der Winkel zwischen beiden Augenstielen ca. $110^{\circ}$.

Die Linse ist kugelig geformt, der Durchmesser beträgt ca. 0.15 $\mathrm{mm}(7,5 \mathrm{~mm})$.

Der Augenstiel verläuft beinahe geradeaus und verbindet sich mit dem unteren Teil des Augenbechers.

Mikroskopisch: Die Kornea besteht aus zwei Zellenschichten. Die Substantia propria ist nur spurweise sichtbar. Die Innenfläche der Hinterwand der Linse beginnt gegen den Linsenblasenraum eine leichte Vorwölbung zu bilden, wodurch auf Schnitten der Linsenraum eine Neumondform zeigt. Die linse steht von der Retina und dem vorderen Rand des Augenbechers noch zienlich weit entfernt.

Das Retinalblatt besteht aus mehreren Zellenschichten. Das Pigmentblatt enthält noch ganz spärliche Pigmentkörner.

Der schmale Sehventrikel kommuniziert durch den Augenstielkanal mit dem Hirnventrikel.

4) Stadium 4 (20 $\mathrm{mm}$ lange Larve).

Die Riechgrube ist stark vertieft. Die Septumbildung für die Bogengänge hat gerade begonnen.

Das Auge (Fig. 11,12) ist etwas grösser geworden als beim vorigen Stadium.

Die Masse der beiden Augen:

Das linke Auge.

Der dorsoventrale Durchmesser

Der nasotemporale Durchmesser

Der anteroposteriore Durchmesser

Die Länge des Augenstiels

Die Dicke resselben

Das rechte Auge.

Der dorsorentrale Durchmesser

Der nasotemporale Durchmesser

Der anteroposteriore Durchmesser

Die Länge des Augenstiels

Die Dicke rlesselben

ca. $0,56 \mathrm{~mm}(28 \mathrm{~mm})$,
ca. $0,44 \mathrm{~mm}(22 \mathrm{~mm})$,
ca $0,38 \mathrm{~mm}(19 \mathrm{~mm})$,
ca. $0,40 \mathrm{~mm}(20 \mathrm{~mm})$,
ca. $0,06 \mathrm{~mm}(3 \mathrm{~mm})$,
ca. $0,56 \mathrm{~mm}(28 \mathrm{~mm})$,
ca. $0,48 \mathrm{~mm}(24 \mathrm{~mm})$,
ca. $0,40 \mathrm{~mm}(20 \mathrm{~mm})$,
ca. $0,38 \mathrm{~mm}(19 \mathrm{~mm})$,
ca. $0,06 \mathrm{~mm}(3 \mathrm{~mm})$. 
Der Winkel zwischen beiden Augenstielen (a. 110 ${ }^{\circ}$.

Der Augenstiel richtet scine leichte Konkavität nach unten aussen.

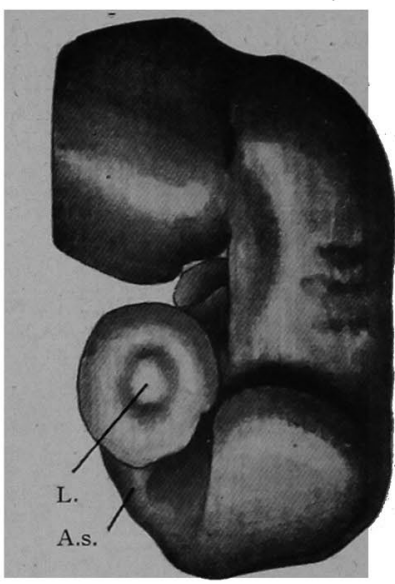

Fig., 11.

Laterale Insicht des Modells des rechten Auges. Stadium 4, 50 mal verriössert modelliert und auf 25 mal verkleinert reproduziert. I. Iinse.

A.s. Augenstiel.

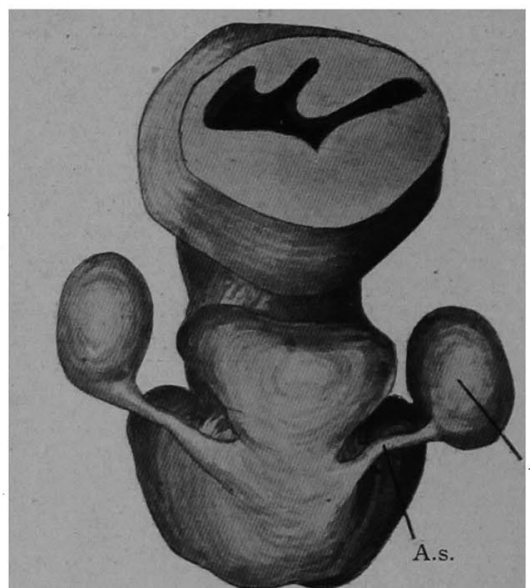

Fig. 12.

Hintere Ansicht desselben Modells. 1. Auge. A.s. Augenstiel.

Die Linse ist kuglig, ihr Durchmesser beträgt ca. $0,16 \mathrm{~mm}$ ( $8 \mathrm{~mm})$.

Mikroskopisch: Die Kornea besteht aus 2 Schichten von Plattenzellen. Die Substantia propria ist noch spurweise zu erkennen. Bezüglich der Linse sieht man keine nennenswerten Veränderungen im Vergleich zum vorigen Stadium. Die Innenflächo ihrer Hinterwand wölbt sich hügelartig in den Blasenraum vor, wodurch er schmäler wurde als vorher.

Das Retinalblatt ist viel dicker creworden, sodass der Alstand zwischen demselben und der Linse etwas geringer ist als vorher. Der Rand des Augenbechers steht noch ziemlich weit ron der Linse entfernt. Das Pigmentblatt zeigt keine merklichen Veränderungen. Inncrhalb des Augenstiels sieht man noch den ganz schmalen Kanal, der mit dem Hirnventrikel kommuniziert. Chondroblasten in der Seitenwand des Schädels kann man noch nicht vorfinden.

5) Stadium $5(20,5 \mathrm{~mm}$ lange Larve).

Die laterale Bogentasche ist nach aussen halbzylindrisch vorgewölbt. 
Die Masseder beiden Augen (Fig. 13,14) sind:

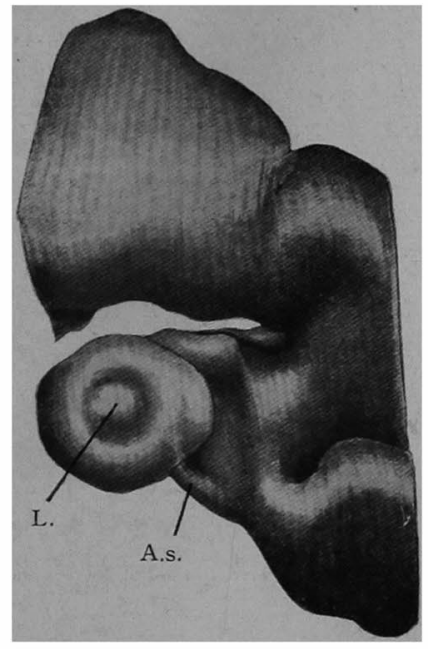

Fig. 13.

Laterale Ansicht des Modells des rechten Auges. Stadium 5, $50 \mathrm{mal}$ vergrössert modelliert und auf $25 \mathrm{mal}$ verkleinert reproduziert. I. Iinse A.s. Augenstiel.

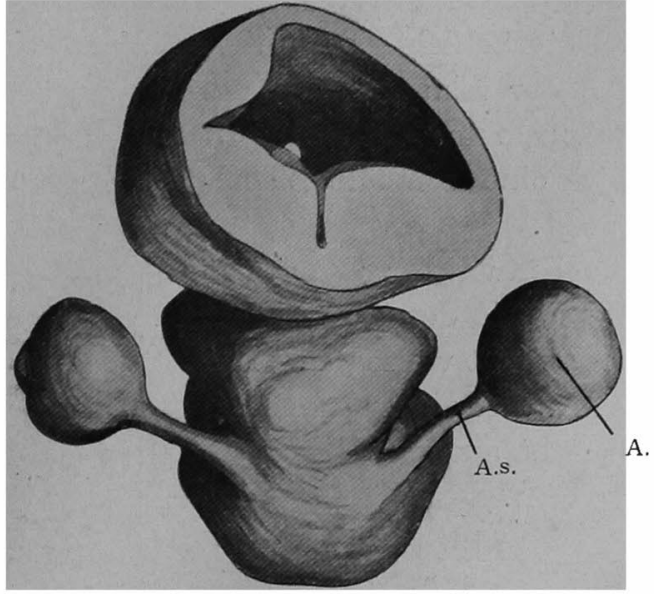

Fig. 14.

Hintere Ansicht desselbem Irodells. A. Auge. A.s. Augenstiel.

Das linke Auge.

Der dorsoventrale Durchmesser

ca. 0,54 $\mathrm{mm}(27 \mathrm{~mm})$,

Der nasotemporale Durchmesser

ca. $0,48 \mathrm{~mm}(24 \mathrm{~mm})$,

Der anteroposteriore Durchmesser

(a) $0,46 \mathrm{~mm}(23 \mathrm{~mm})$,

Die Länge des Augenstiels

Die Dicke desselben

ca. $0,44 \mathrm{~mm}(22 \mathrm{~mm})$,

ca. $0,06 \mathrm{~mm}(3 \mathrm{~mm})$.

Das rechte Auge.

Del: dorsoventrale Durchmesser

Der nasotemporale Durchmesser

ca. $0,56 \mathrm{~mm}(28 \mathrm{~mm})$,

ca. $0,56 \mathrm{~mm}(28 \mathrm{~mm})$,

Der anteroposteriore Durchmesser

ca. $0,44 \mathrm{~mm}(22 \mathrm{~mm})$,

Die Länge des Augenstiels

ca. 0,44 $\mathrm{mm}$ (22 $\mathrm{mm})$,

Die Dicke desselben

ca. $0,06 \mathrm{~mm}(3 \mathrm{~mm})$.

Der Winkel zwischen beiden Augenstielen ca. $120^{\circ}$.

Der Augenstiel richtet seine leichte Konkavität nach aussen unten. Der Stielkanal ist ganz schmal geworden, ist aber noch durchgängig.

Mikroskopisch: In diesem Stadium zeigt sich die Linse nicht als kuglig, sondern als etwas ellipsoidisch (Fig. 15). Der durchschnittliche 
Durchmesser beträgt ca. $0,16 \mathrm{~mm}(8 \mathrm{~mm})$. Ihr anteroposteriore Durchmesser ist etwas länger als die sonstigen Durchmesser. Die im Linsenraum vorgewölbte Schicht der hinteren W'and nimmt bedeutend an Grösse zu, wodurch der Linsenraum ganz schmal wurde und auf Schnitten sich als eine schmale Sichelform zeigt.

Die Entwicklung der Substantia propria der Kornea hat sich noch nicht vollzogen. Das Retinalblatt ist dicker geworden und besteht aus mehreren Zellenschichten. Das Pigmentblatt ist in diesem Stadium ziemlich stark pigmentiert. In dem Gewebe der Umgebung des Pigmentblattes sieht man ebenso auch reichliche Pigmentkörner.

6) Stadium $6 \quad(23,5 \mathrm{~mm}$ lange Larve).

Dic Nasenhöhle ist röhrenförmig verlängert. Die vordere Bogentasche ist beinalle abgesch-

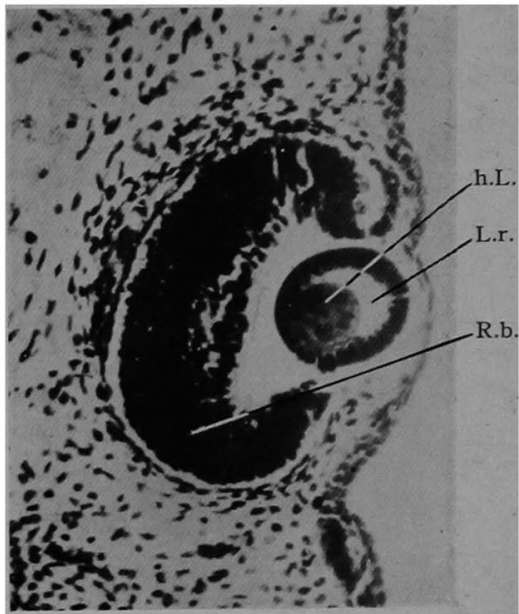

Fig. 15).

Frontaler Durchschnitt des rechten Auges (Stadium 5, Vergr. ca. 80 mal). I.r. Linsenraum. h.J. Hintere Linsenwand. R.b. Retinalblatt. nürt.

Die Masse der beiden Augen (Fig. 16,17) sind:

Das linke Auge.

Der dorsoventrale Durchmesser

Der nasotemporale Durchmesser

Der anteroposteriore Durchmesser

Die Länge des 'Augenstiels

Die Dicke resselben

Das rechte Auge.

Der dorsoventrale Durchmesser

Der nasotemporale Durchmesser

Der anteroposteriore Durchmesser

Die Länge des Augenstiels

Die Dicke desselben ca. $0,60 \mathrm{~mm}(30 \mathrm{~mm})$, ca. $0,58 \mathrm{~mm}(29 \mathrm{~mm})$, ca. $0,52 \mathrm{~mm}(26 \mathrm{~mm})$, ca. $0,60 \mathrm{~mm}(30 \mathrm{~mm})$, (a. $0,06 \mathrm{~mm}(3 \mathrm{~mm})$.

ca. $0,60 \mathrm{~mm}(30 \mathrm{~mm})$, ca. $0,60 \mathrm{~mm}(30 \mathrm{~mm})$, (a. $0,50 \mathrm{~mm}(25 \mathrm{~mm})$, ca. $0,60 \mathrm{~mm}(30 \mathrm{~mm})$, ca. $0,06 \mathrm{~mm}$ ( $3 \mathrm{~mm})$.

Der Winkel zwischen beiden Augenstielen. ('a. $130^{\circ}$.

Der Augenstiel richtet seine Konkavität nach aussen unten, die viel 
stärker als beim vorigen Stadium ist. Dio Verbindungsstelle des Augensticls mit der Augenbecherwand ist hier etwas nach oben rerschoben.

Der Durchmesser der Linse beträgt durchschnittlich ca. $0,18 \mathrm{~mm}$ (9 $\mathrm{mm})$; ihre Form ist noch etwas ellipsoidisch.

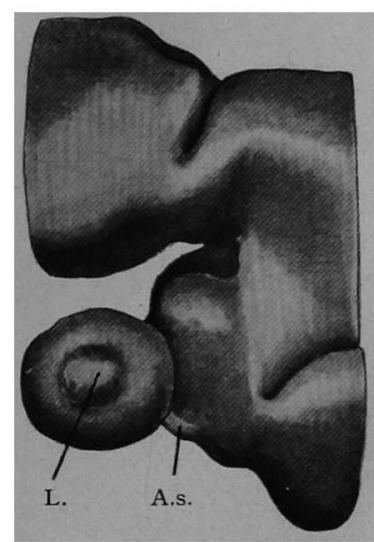

Fig. 16.

Jaterale Ansicht des Modells des rechten Auges. Stadium 6, $50 \mathrm{mal}$ vergrössert modelliert und auf 25 mal verkleinert reproduziert. T. Linse. 1.s. Augensticl.

Mikroskopisch : Die Innenfläche der hinteren Linsenwand ist so stark rorgewölbt, dass sie sich als ein Kügelchen erweist, das aus den Fasern besteht. Der Linsenblasenraum ist mit diesem Kügelchen fast vollständig ausgefüllt und so bleibt nur eine ganz schmale Lücke übrig.

Die Kornea besteht aus 2 Schicht'n von Plattenzellen. Der Entwicklungszustand der Substantia propria ist beinahe dergleiche wie vorher. Das Retinalblatt ist dick und lässt erst drei Schichten aus zelligen Elementen unterscheiden (Fig. 18) (Nervenzollen-, innere und äussere Körnerschichten). Das Pigment-

blatt zeigt keine veränderungen. In diesem Stadium findet man zuerst die Nervenfaserbildung (Fig. 19) in der Augenstielwand. Auch sieht man hier zum erstenmal die Bildung des Chondrokraniums.

7) Stadium $7(26,5$ mm lange Larve).

Die Nasenhöhle kommuniziert durch die Choana mit der Mundhöhle. Der vordere Bogengang ist schon vom Utriculus vollsändtig abgeschnürt.

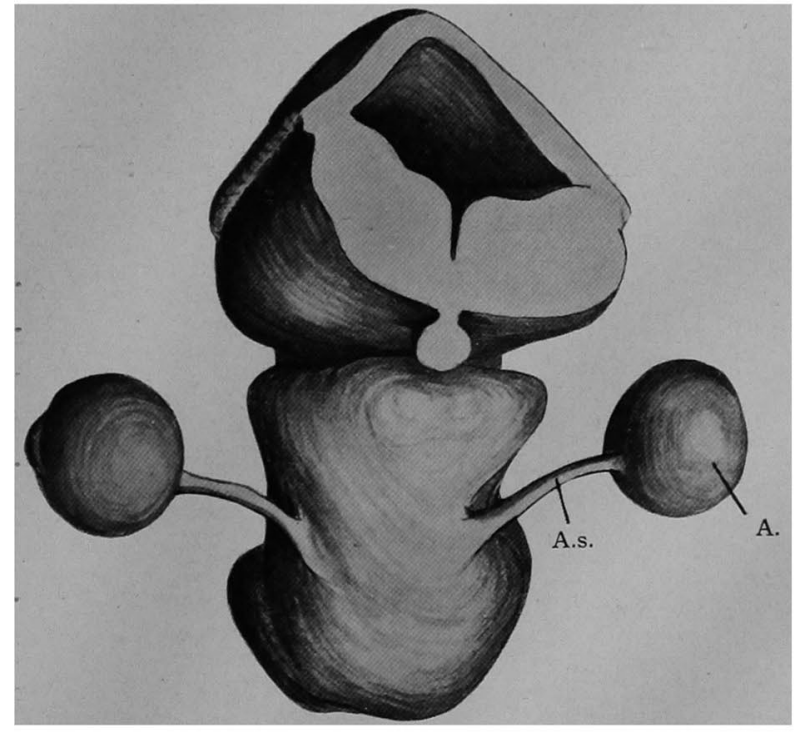

Fig. 17.

Hintere Ansicht desicllen Modells. A. Auge. A.s. Augenstiel. 
In cliesem Stadium ist das Wachstum des Auress (Fig. 20, 21) ganz boleutend zu erkenn'm.

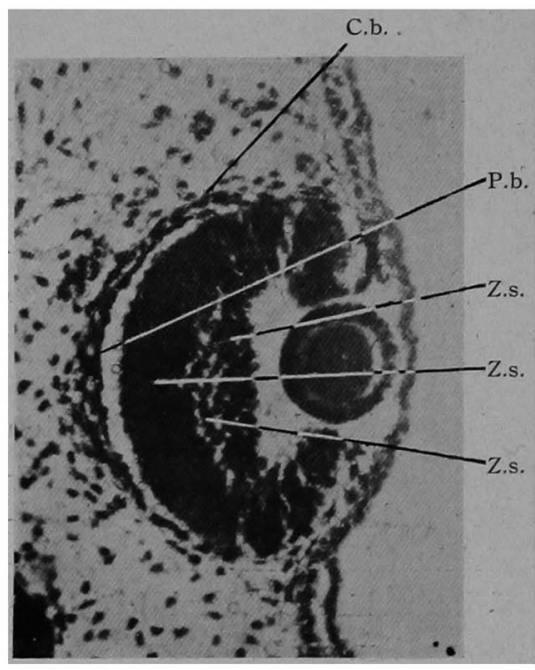

Fig. 18.

Frontaler Durchichnitt des rechten Auges. (Stadium 6, Verer. ('a. 80 mal). ('.). Chorioidealblatt. P.b. Pigmentblatt. Z.s. Zellenschicht.

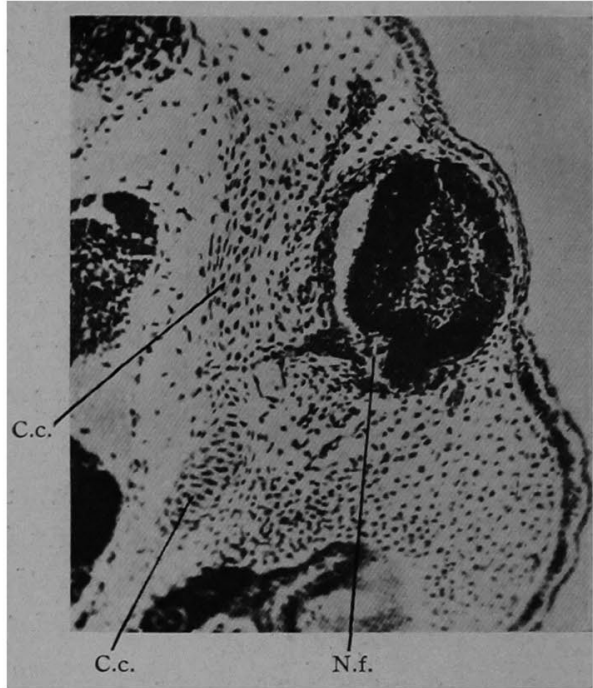

Fig. 19.

Frontaler Durchschnitt des rechten Auges. (Stadium 6, Vergr. ca. 45 mal). N.f. Nervenfasern. (C.c. Chondrocraninm.

Dir Masse der beiden Augen sind:

Das linke Auge.

Der dorsoventrale Durchmesser

Der nasotemporale Durchmesser

Der anteroposteriore. Durchmesser

Die Länge des . Iugenstiels

Die Dicke resselben

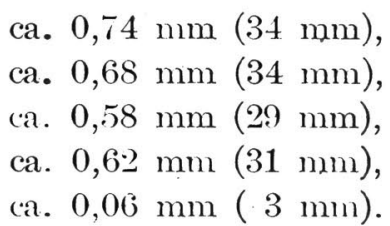

ca. $0,72 \mathrm{~mm}(36 \mathrm{~mm})$, (a. $0,68 \mathrm{~mm}(34 \mathrm{~mm})$, (a. $0,58 \mathrm{~mm}(29 \mathrm{~mm})$, (a. $0,62 \mathrm{~mm}(31 \mathrm{~mm})$, (a. $0,06 \mathrm{~mm}(3 \mathrm{~mm})$.

Die Dicke resselben

Der W'inkel zwischen boirlen Augenstielen . (a. $130^{\circ}$. 


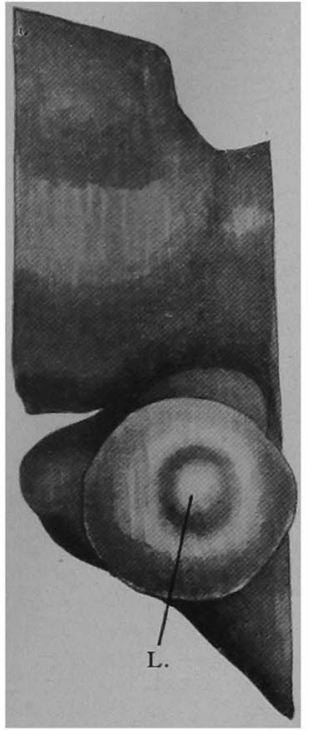

Fig. 부.

Iaterale Ansicht des Modells des rechten Auges. Stadium 7, $50 \mathrm{mal}$ vergrössert modelliert und auf $25 \mathrm{mal}$ verkleinert reproduziert.

Der Augenstiel verläuft nach aussen oben, mit der Konkavität nach aussen unten gerichtet; seine Krümmung ist äusserst stark geworden. Seine Terbindungsstelle mit dem Augenbecher hat sich bedeutend weiter zur Mitte des letzteren verschoben. Der Durchmesser der Linse bcläuft sich auf ca. $0,2 \mathrm{~mm}(1 \mathrm{~mm})$, und ihre Gestalt ist wieder kuglig geworden.

Mikroskopisch: Die Kornea zeigt keine beträchtlichen Veränderungen verglichen mit dem vorigen Stadium. Die Linse ist sehr gut ent-

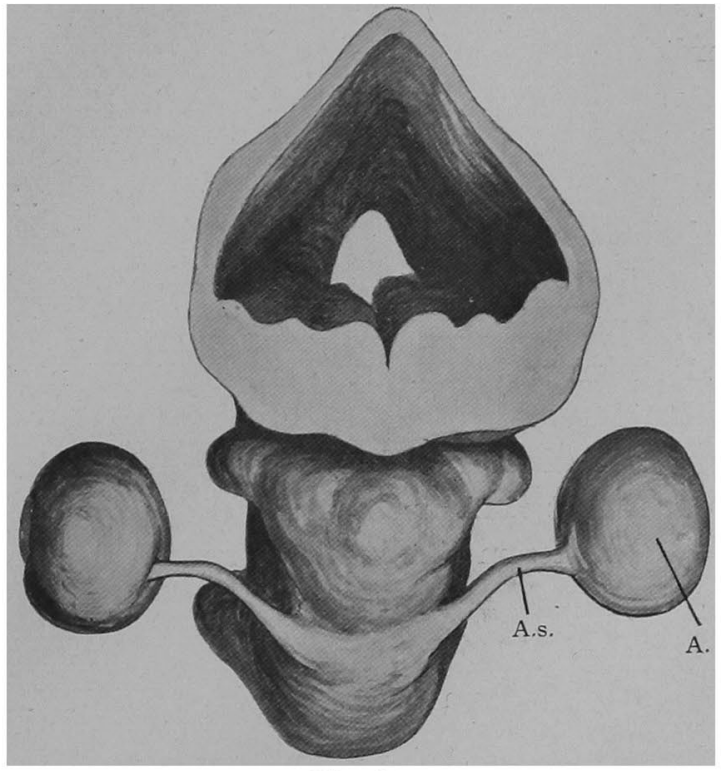

Fig. 21.

Hintere Ansicht desselben Modells.

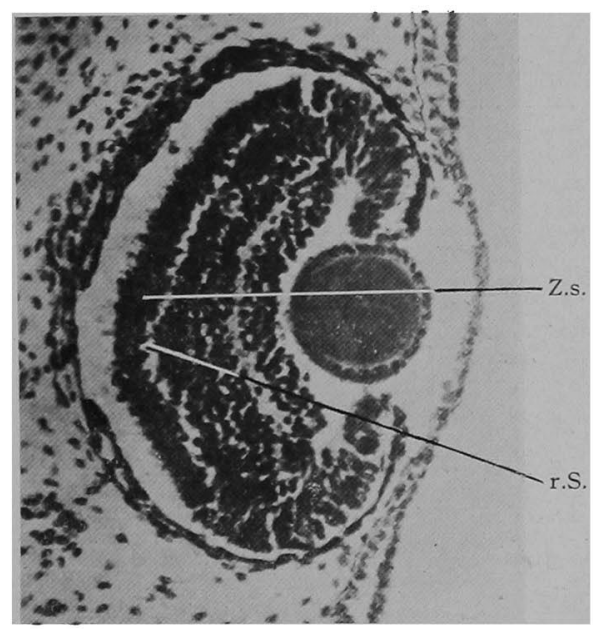

Fig. 22,

Frontaler Durchschnitt des rechten Auges. (Stadium 7, Vergr. ca. 80 mal). Z.s. Zellenschicht. r.S. retikuläre Sichicht. 
wickelt und die Innenschicht ihrer Hinterwand ist nach innen stark hervorgetrieben. Die Distalfläche dieser Wand steht, beinahe in Berührung mit der Proximalfäche der Vorderwand derselben. Die Retina und der Augenbecherrand haben sich gegen die Linse genähert. Das Retinalblatt hat an Dicke zugenommen, und die Differenzierung der drei Zellenschichten ist viel deutlicher geworden (Fig. 22). Die retikulären Schichten sind auch erkemmbar und die inmere retikuläre ist schon viel breiter als die äussere. Das Pigmentblatt enthält Pigmentkörner und besitzt im allyemeinen ein bräunliches Aussehen. Die Chorioidea ist ebenso auch stark pigmentiert. Der Augenstiel enthält noch ein ganz schmales Kanälchen.

Die Knorpelbildung d Schädelseitenwand ist äusserst fortgeschritten, doch sieht man noch das weit offen gebliebene Foramen opticum.

In diesem Stadium kann man noch keine Spur des Skleralknorpels vorfinden.

\$) Stadium $8(30 \mathrm{~mm}$ lange Larvo).

Die Masse beider Augen sind:

Das linke Auge.

Der dorsoventrale Durchmesser:

ca. $0,75 \mathrm{~mm}(49 \mathrm{~mm})$,

I) (er nasotemporale Durchmesser

ca. $0,81 \mathrm{~mm}(53 \mathrm{~mm})$,

Der anteroposteriore Durchmesser

ca. $0,68 \mathrm{~mm}(45 \mathrm{~mm})$,

Die Länge des Augenstiels

ca. $1,00 \mathrm{~mm}$,

Die Dicke desselben

ca. $0,06 \mathrm{~mm}(4 \mathrm{~mm})$.

Das rechte Auge.

Der dorsoventrale Durchmesser

Der nasotemporale Durchmesser

Der anteroposteriore Durchmesser

Die Länge des Augenstiels

ca. $0,75 \mathrm{~mm}(49 \mathrm{~mm})$,

ca. $0,78 \mathrm{~mm}(51 \mathrm{~mm})$,

ca. $0,74 \mathrm{~mm}(48 \mathrm{~mm})$,

ca. $1,00 \mathrm{~mm}$,

Die Dicke desselben

ca. $0,06 \mathrm{~mm}(4 \mathrm{~mm})$.

Der Durchmesser der Linse beträgt ca. $0,3 \mathrm{~mm}(2,0 \mathrm{~mm})$. In der Skleralwand der beiden Augen findet man bereits drei und vier Stücke les Skleralknorpels. Auf die einzelnen Knorpelstücke werde ich später noch näher eingehen.

Mikroskopisch: In diesem Stadium hat der Augenstiel seine Stielhöhle verloren und ist vollständig von Nervenfasern eingefasst (Fig. 23). Der Nervus opticus dringt in den Augenhintergrund fast in der Mitte der medialen Seite des Bulbus ein. 
Die Kornea besteht aus einer oberen kubischen- und einer unteren platten Zellenschicht. Die Substantia propria ist ziemlich gut entwickelt und stellt eine dünne Schicht mit spärlichen Plattenzellen dar. Die Epithelzellen der Vorderwand der Linse sind kubisch, doch je seitlicher sie stehen, desto länger werden sie und in der Nähe des hinteren Pols nehmen sie schliesslich eine vollkommen zylindrische Form an. Die Zellkerne liegen in der hinteren Hälfte der Linsenfasern und bilden zusammen eine Kernzone.

Die Retina hat bedeutend an Dicke zugenommen und dadurch nähert sie sich der Lins', so dass sie mit dem proximalen Ende der Linsenwand beinahe in Berührung zu stehen scheint. Die innere retikuläre Schicht und Sehzellenschicht sind deutlich differenzicrt.

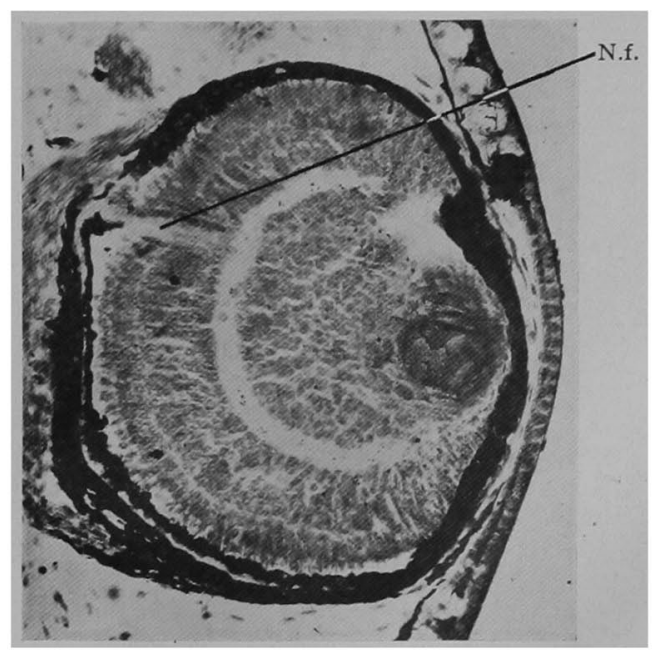

Fig. 23.

Frontaler Durchschnitt des rechten Auges. (Stadium 8, Vergr. ca. $80 \mathrm{mal}$ ). N.f. Tervenfasern.

Die Iris scheint beinahe

völlig ausgebildet $\mathrm{zu}$ scin, sie ist so stark pigmentiert, dass sie ganz schwärzlich aussieht, und steht mit der Linse in Berührung. Die Pigmentepithelzellen haben reichliche Pigmentkörner, besonders in der Nähe der Aussenfläche. Die Chorioidea enthält die länglichen platten Pigmentzellen und in der Nähe ihrer Aussenfläche findet man cinige kleine Stücke vom auftretenden Skleralknorpel. Alle Knorpelstücke sind von den Chorioidealzellen umhüllt.

Das Chondrokranium ist äusserst gut entwickelt, doch steht das Foramen opticum noch ziemlich weit offen.

Das V'orkommen des Skleralknorpels.

In diesem Stadium findet man zuerst drei und vier kleine Stücke von Skleralknorpel vor (Fig. 24, 25). In Bezug auf ،lie Stellen seines ersten Auftretens glaube ich, dass eine bestimmte Regel herrscht, weil er an beiden Augen ganz symmetrisch vorkommt. Im folgenden werde ich auf die Modelle etwas näher eingehen. 
Das linke Auge (Modell 8).

Am linken Auge sieht man 3 Knorpelstücke, die alle im allgemeinen längliche Form haben und im ganzen um den Opticus herum fast radiär stehen. Die Eintrittsstelle des Opticus in die Skleralwand liegt heinahe in der Mitte der medialen Bulbuswand.

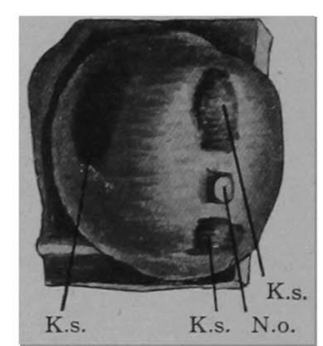

Fig. 24.

Hintere Ansicht des Modell: des linken Auges. Stadium 8, ca. tit mal vergrössert modelliert und auf :i: mal verkleinert reproduziert. K.s. Knorpelstï̈ck. N.o. Nerrus opticus.

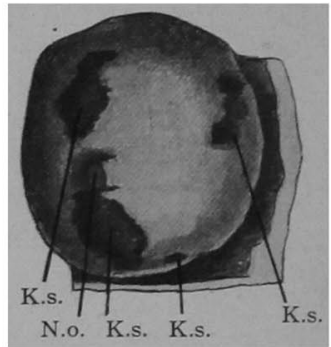

Fig. :5.

Hintere Ansicht des Modells des rechten luges. Stadium 8,66 mal vergrössert modelliert und auf 33 mal verkleinert reproduziert.

a) Das erste Stück befindet sich nasal rom Opticus, ca. 0,03 $\mathrm{mm}$ davon entfernt ; sine Längsachse steht fast horizontal in anteroposteriorer Richtung. Das Stück weist folgende Masso auf :

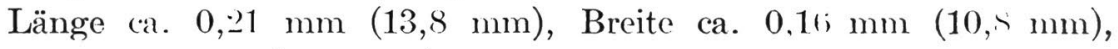
Dicke (a. 0,05 $\mathrm{mm}(0,35 \mathrm{~mm})$.

'b) Das zweite Stück liecet dorsal und etwas naral roni Opticus, seine Längsachse steht schräg von hinten oben nach vorn unten gerichtet. Dic Aussenfläche des Knorpelstückes ist mit ziemlich dicker chorioidealer Pigmentzellenschicht überzogeu. Sein hinteres Ende licert dorsal ca. $0,4 \mathrm{~mm}$ rom Opticus entfernt. Die Masio sind :

Länge (a). $0,27 \mathrm{~mm}$ (17,\$ mm), Breite ca. $0,21 \mathrm{~mm}$ (13,i mm), Dicke ca. $0,06 \mathrm{~mm}(4,0 \mathrm{~mm})$.

c) Das lritte Stück reprïsentiert eine kurze Platte und lingt gerade temporal rom Opticus, sein Nasalende steht ca. 0,06 mm rom Opticus entfernt. Die Masse des Stïckess sind:

Läığ ca. 0,12 mm $(7,9 \mathrm{~mm})$, Breite ca. $0,21 \mathrm{~mm}(14,0 \mathrm{~mm})$, Dicke ca. $0,07 \mathrm{~mm}(-1,6 \mathrm{~mm})$.

Das rechte Auge (Modell 8).

Die Eintrittsetelle des Opticus ist beinahe gleich wie beim linken Auge. 
In rechten Auge findet man 4 Knorpelstücke.

a) Das erste Stück liegt nasal vom Opticus und steht mit der Längsachse fast horizontal, von hinten unten nach vorn oben wenig gencigt. Scin hinteres Ende steht ca. 0,06 mm rom Opticus entfernt. Dic Masse sind:

Länge'ca. 0,3 mm (19,8 mm), Breite ca. $0,18 \mathrm{~mm}(11,8 \mathrm{~mm})$, Dicke ca. $0,05 \mathrm{~mm}(3,3 \mathrm{~mm})$.

b) Das zweite Stück befindet sich an dorsaler und etwas nasaler Seite des Opticus. Seine Längsachse steht von hinten unten nach vorn oben gerichtet. Das hintere Ende steht ca. $0,3 \mathrm{~mm}$ vom Opticus entfernt.

Länge ca. $0,27 \mathrm{~mm}(17,8 \mathrm{~mm})$, Breite ca. $0,12 \mathrm{~mm}(0,8 \mathrm{~mm})$, Dicke ca. $0,05 \mathrm{~mm}(3,3 \mathrm{~mm})$.

c) Das dritte Stück liegt gerade an temporaler Seite des Opticus

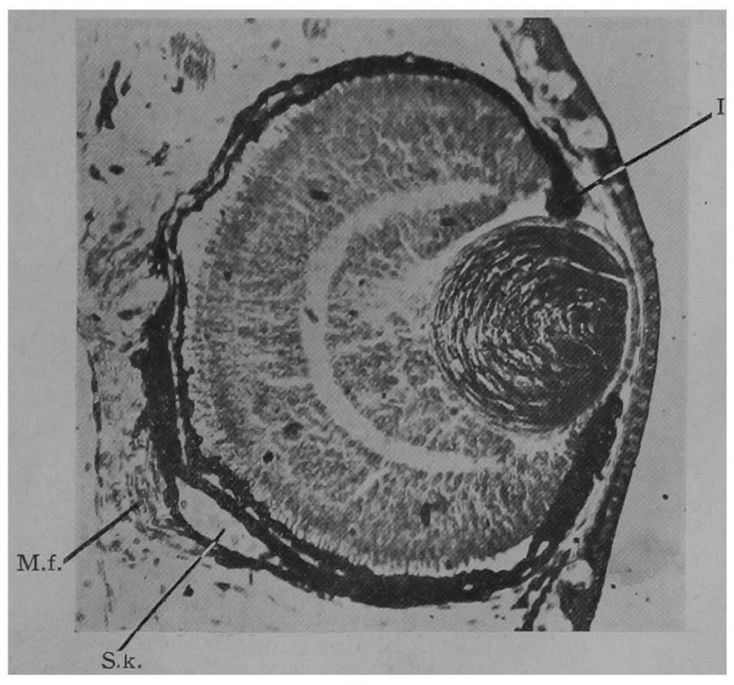

Fig. 26.

Frontaler Durchichnitt des rechten Auges. (Stadium s, Vororr. ca. $80 \mathrm{mal}$ ). I. Iris.

s.k. Skleralknorpel. M.f. Muskelfasern. und seine Längsachse steht ein wenig von vorm unten nach hinten oben gerichtet.

Länge ca. $0,24 \mathrm{~mm}$ $(15,4 \mathrm{~mm})$, Breite ca. $0,23 \mathrm{~mm}(15,2 \mathrm{~mm})$, Dicke ca. $0,06 \mathrm{~mm}$ $(4,0 \mathrm{~mm})$.

d) Das vierte Stück ist das kleinste, dessen anteroposteriorer Durchmesscr nur ca. 0,03 $\mathrm{mm}$, der dorsoventrale ca. $0,08 \mathrm{~mm}$ und die Dicke ca. 0,06 $\mathrm{mm}$ beträgt. Dieser kleine Knorpelklumpen liegt temporodorsal rom Opticus sehr weit davon entfernt.

Mikroskopisch: Die Skleralwand verdickt sich an den Stellen, wo dic Knorpelstücke aufgetreten sind. Der Skleralknorpel ist ziemlich reichlich mit hyaliner Grundsubstanz rersehen; die Form der Knorpelzellen ist entweder rundlich oder oval und die Anordnung der Zellen ist ganz unregehmässig. Die Zelle besitzt einen ziemlich grossen Kern. In diesem Stadium haben sowohl dio Knorpelzellen als auch die Grund- 
substanz noch keine Pigmentkörner. Auf Schnitten sieht man ohne Ausnahme, lass die Augenmuskelfascrn an der Oberfläche des Knorpelstückes sich anset\%(n (Fig. 26).

9) Starlium 9 (80 mm langes Exemplar).

Das rechite Auge hat folgende Masse:

Der dorsoventrale Durchmesser

ca. $1,66 \mathrm{~mm}(110,0 \mathrm{~mm})$,

Der nasotemporale Durchmesser

(a. $1,36 \mathrm{~mm}(89,8 \mathrm{~mm})$,

Der anteroposteriore Durchmesser

(a. $1,24 \mathrm{~mm}(81,8 \mathrm{~mm})$,

- Die Iü̈nge des Opticus

Die Dicke llsselben

(a. $2,0 \mathrm{~mm}$

Der Durehnesere der Linse

(a. $0.1 \mathrm{~mm}(6,6 \mathrm{~mm})$, (a. 0,$6 ; 4 \mathrm{~mm}(42,20 \mathrm{~mm})$.

Der äusserst grut ('ntwickelte Skleralknorpel wrist schon in diesem Starlium einen rollkommenen Knorpelbecher auf (Fig. 27). Seine Wand ist ziemlich dick und liegt auf dem grösiten Teil der hinteren Hälfte dus Augapfels ausgebreitet.

Fast in der Mitte des Becherbodens findet man cinen äusserst grossen Kanal (Canalis opticus), dessen Rand uneben ist, wennmgleich man hier woller Knorpelfortsätze noch Knorpelinseln vorfinden kann. Der Kanal int beinahe rundlich, hat einen Durchmesser von ca. 0.3 cm (2 cm) und dient zum Durchtritt sowohl des Sehmerven als

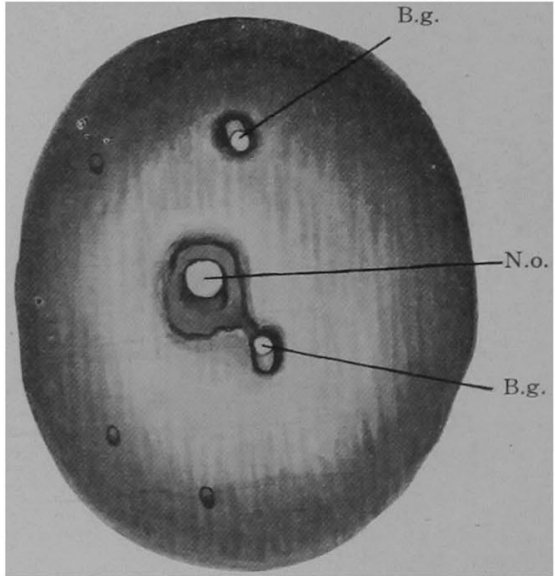

Fig. 27.

Proximale Ansicht des Modells des rechten Auges. Starlium !. $66 \mathrm{mal}$ verer:iswert molelliert und auf $: 3: 3$ mal verkleinert reproduziert. X.o. Yer-

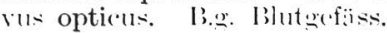
auch der Blutgefäise. Der Opti(us macht eine leichte Biegung, bevor er in die Netzhaut eintritt (Fig. 2s). Von dissem Kanal verzweigt sich in ventrotemporaler Richtung ein feiner G(fäiskanal (ca. 0,1 mm weit). Auch sicht man oben und etwas hinten vom Opticuskanal, davon (a. $0,25 \mathrm{~mm}$ entfernt, ein klcines Gefässkanälchen mit einem Durchmesser von ca. 0.15 mm. Noch ein kleines, $0,06 \mathrm{~mm}$ weites Greä̈skanälchen befindet sich an der dorsalen Seite des Opticuskanals, davon etwa $0,35 \mathrm{~mm}$ cutfernt. 
Ausscrelem findet man noch 3 ganz kleine Kanälchen; das einc stcht temporal rom Opticuskanal, ca. 0,3, $\mathrm{mm}$ davon entfernt, das zweite rentral rom lotyteren, a. $0,53 \mathrm{~mm}$ davon entfernt und cndlich befindet sich das dritte an ventronasaler Scite, ca. $0,44 \mathrm{~mm}$ davon

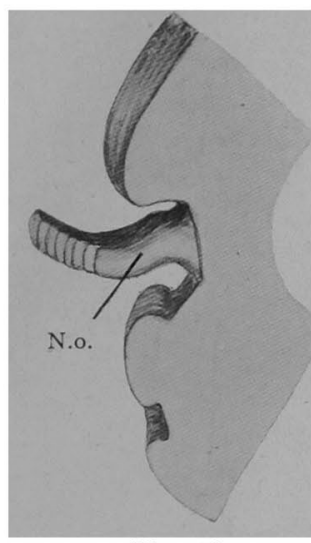

Fig. 오.

Dir. Insiclat des Modells des sohnerren, das die Verhältnirse seiner Biegung zeigt. N.o. Xorvu. opticus. (ntfernt. Die Becherwand ist an dicksten am Boden und verdünnt sich allmählich nach allen S'iten hin. Sir endigt mit einem keilförmigen spitzen Rand. Die Gestalt des Knorpelbechers ist nicht rundlich, sondern ellipsoidisch in senkrechter Richtung verlängert, so lasss sein nasotemporaler Durchmesser ea. 1,3 mm und der dorsoventrale $1,(6 \mathrm{~mm}$ bet räigt ; scine grösste Dicke beläuft sich auf ca. 0,12 mm.

Mikroskopisch: I)(r Sklcralknorpel (Fig. 29) bestcht aus hyaliner Grundsubstanz und relativ reichlichen Knorpelzellen, die zweierlei Formen aufweisen, eine rundliche und eine ovale. Manche von diescon Zellen und die Grundsubstanz onthalton eine klcine Menge von Pigmentkörnern. Die Knorpelzollen sind durch die gesante Dicke dre Knorpols hindurch in ganz vorschiedenen Richtungen angeordnct. Im Vergleiche mit den vorigen Stadium sind die Zellkerne bedentend vorkleinert, wodurch die Zellen ziemlich protoplasmareich wurden. Dis Form der Kerne ist whr verschicden, rundlich, länglichrund, halbmondförmig und dreieckig.

Die Linsc ist rundlich, das Epithel besteht aus einschichtigen Plattenzell'n, aber je woiter wir seitlich kommen, desto längre werken sie. Dir Kerne bilden boinahe in mittleren Teil der Linsonfasern eine Kernzone.

Die Retina ist jetzt äusserst wut cntwickelt, indem jede zellige S.hicht aus einigen Zellonlagen besteht, so die änssere Körnerschicht aus 2-3, die immere aus 3-4 und dis Nervenzellenschicht aus 1-2 Zellenschichten. Jetzt kann man auscer den Zell-chichten auch noch die Nervenfascrschicht klar unterscheiclen. Dis Pigmentepithelschicht zeigt sich als brännlich-schwarz; die Pigmentkörner kommon meistens in der Nähe rler Aussenfläche vor.

Die Chorioidea ist ebenfalls stark pigmontiert un!l verdickt sich an den Stellen, wo sie ron Blutgefässon durchzogen wird. Dis Iris ist anch grut entwickelt und berührt dicht die Linse. 


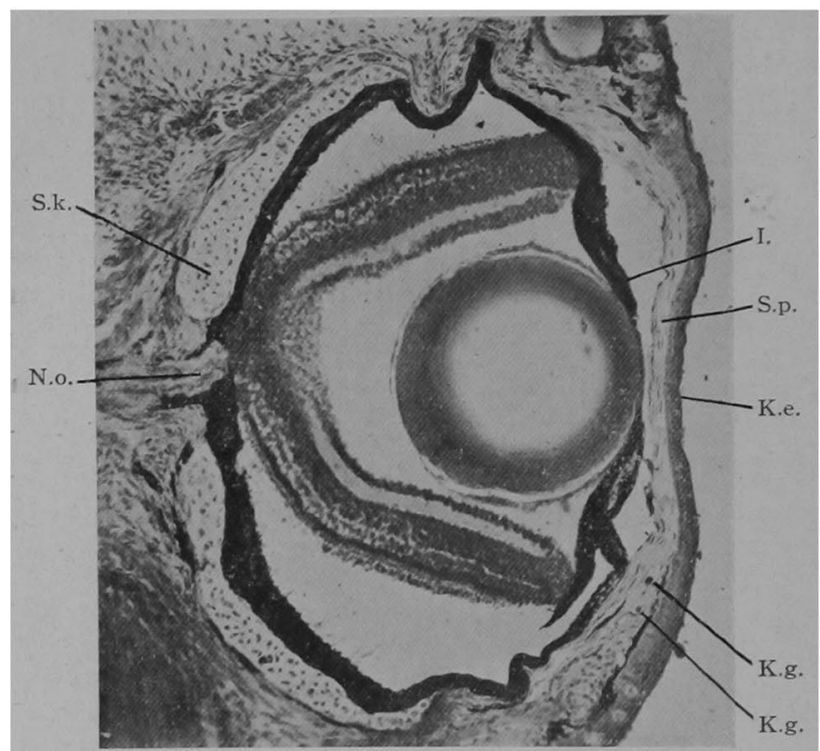

Fig. :).

Frontaler Durelechnitt des rechten Auges (Starlium $\dot{9}$. Vergr. ('a. 45 mal). S.k. Skleralknorpel. N.o. Nervus opticus:

I. Iris. K.g. Kornealgefäise. S.p. Substantia propria K.e. Kornealepithel.

man in der Substantia propria schon dis einfache Verteilung der Blutkapillaren.

Die frühzeitige Hornhautrakkularisation.

Wenn man das Moriell 9 (Fig. 30) betrachtet, so findet man die Blutkapillaren, die rom peripherischen Lmkreis der Hornhant in die letztere eintreten und danach entlang dre Randzone bogenförmig vrlaufen. Dieser äusere (iofüssing ist an der temporalen Seite auf cine kur\% Strecke unterbrochen. Innen davon sieht man noch einen damit parallel ziehenden, ebenfalls temporal ein wenig unterbroch'nen kleinen Gefässring. '/wischen diesen beiden Ringen bestehon zahlreiche Anasto-
Dis Kormea besteht aus 3-4 \%ellenschichten, den oberflächlichen, platten und ticfen kubischen Zellen. Dic Substantia propria ist so verdickt, dass sio fast wleich dick wis die Epithelschicht ist, und enthält spärliche lange platter Zellen; ihre Innenflächı ist nit Enrlothel ülxr. zogen. Im peripherischen Teil der Kornea findet man ureringe Pigmontkörner. In dissem Stadium findet

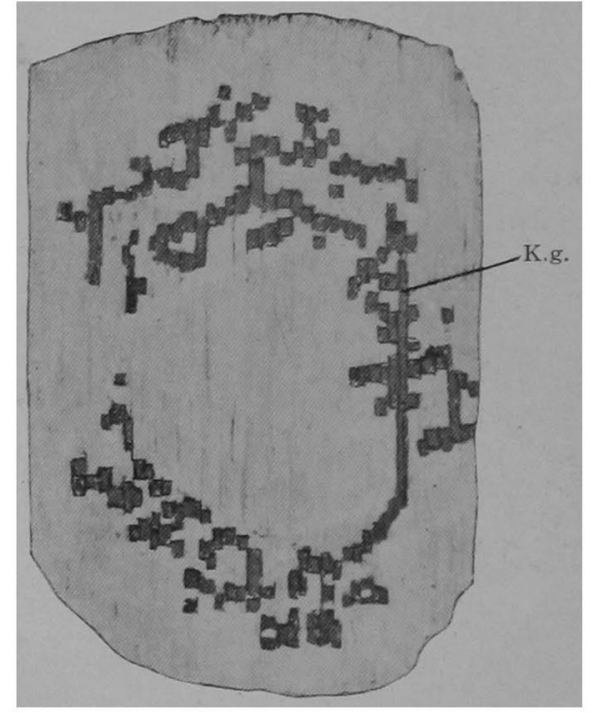

Fig. 30.

Laterale Ansicht des Modells des re.hten Auges. Stadium 9, 66 mal verorisisert. morlelliert und auf 33 mal verkleinert reproduziert. K.g. Komealgefïs:s: 
mosen, wohlurh hier ein ziemlich komplizisetes Randschlingennstz zu stande kommt. In zentralen Teil der Ifornhaut kann man noch keine

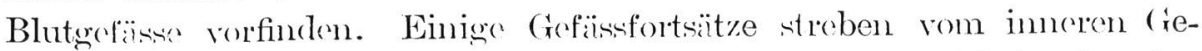
fässing nach der Ifornhautnitte zu. Alle disw befinden sich im dorsilen Teil der Hornhaut und ziehen entwoder nach untem orler nach aussen zu.

Mikrosknpich: In Bozug auf die Lagerung der Kornealgefüsso in

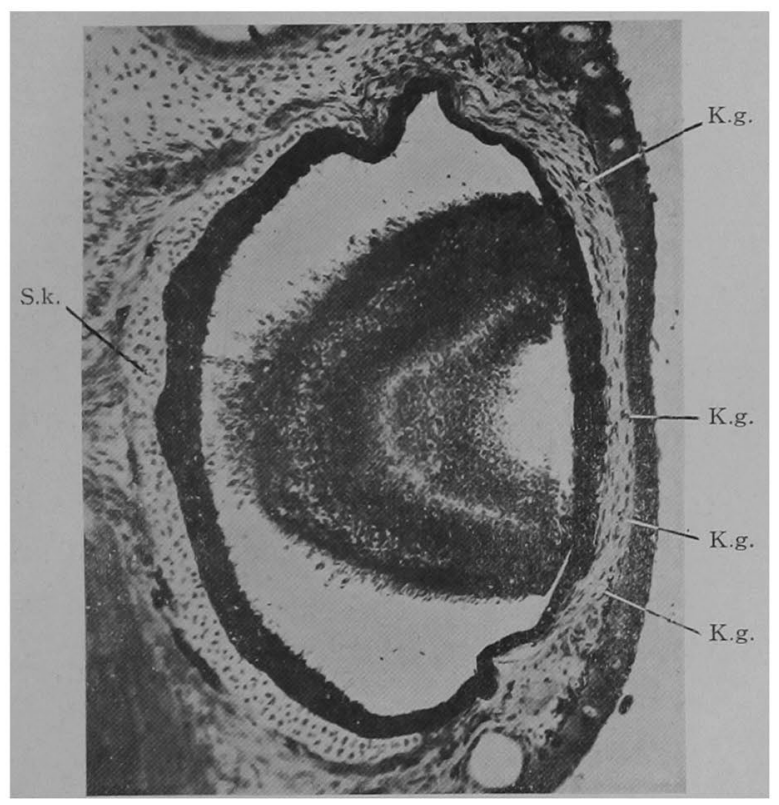

Fig. :31.

Frentaler Durchichnitt des rechten Auges. Standium 9, Voror. ('a. 45 mal). s.k. skleralknorpel. K.⿻. Kiornealgefässce. der Schicht der Substantia propria kann man 2 Arten (Fig. 2?, 31) unterscheiden. Die Kapillaren dor ersten Art liegen im oberflächlichœn Irittel und die der zweiten in den tiefer:n zwei Drittel. Die Wand der Kapillaren ist aus (iner Lage Endothelzellen aufgebaut.

10) Stadium 10 (179 $\mathrm{mm}$ langes Exemplar).

Dicses Exemplar ist las grösste unter deus ron mir untersuchten Tieren, doch ist es noch bedeutend kleince als die bisher von anderen

Autoren untersuchten. Die Grutalt des Bulbus ist eine unregelmässigbirnförmige; die Masse sirul :

Der dorsorentrale Durchnesser.

Der nasotemporale Durchmesier

Der anteroposteriore Durchmesser

Die Länge des Opticus

Die Dicke deswelben

$$
\begin{aligned}
& \text { ca. } 2,0 \mathrm{~mm}(80,0 \mathrm{~mm}) \text {, } \\
& \text { (a. } 2,24 \mathrm{~mm}(98,5 \mathrm{~mm}), \\
& \text { (a. } 2,0 \mathrm{~mm}(80,0 \mathrm{~mm}) \text {, } \\
& \text { (a. } 3,5 \mathrm{~mm}, \\
& \text { a. } 0,11 \mathrm{~mm}(4,4 \mathrm{~mm}) .
\end{aligned}
$$

Der gut entwickelte Knorpelbecher (Fig. 32) hat eine zienlich dicke Wanrl, welche im Bodenteil am stärksten ist, während sie sich nach dem Becherranr zu allmählich verlünnt; sie endet aber nicht auf allen Seiten mit gleicher Dicke, dem dor. Ventrallamel ist viol dicker als der Dorsal- 
rand. Der halbkugelige Becher dehnt sich auf die hinter' Hälfte des Bulbus aus und soin nasotemporaler Durchmesser ist am längsten. Auf dem Becher sicht man '; Kanälchen von verschied(nner W'eite, von den'su sich 5, auf seiner temporalen Mälfte befinden.

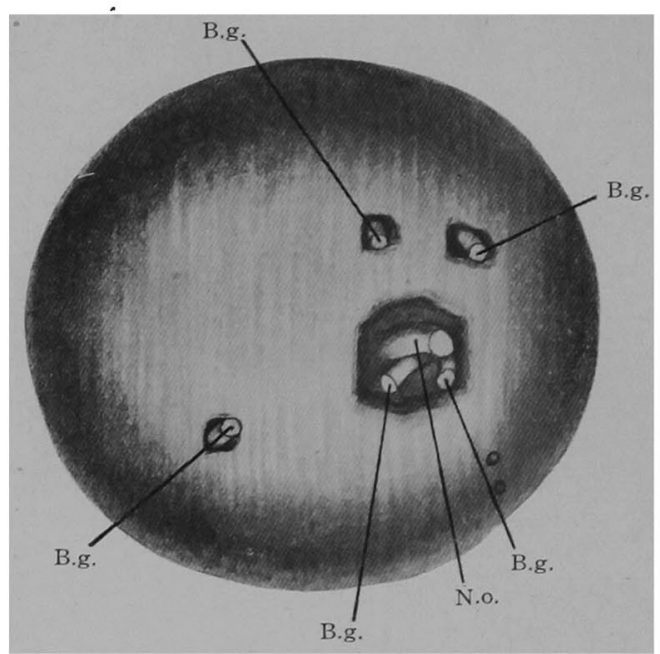

Fì. : :ㄹ.

Proximale Ansicht des Modells des rechten Auges: Starlium 10.41) mal vergrösert modelliert und auf 20 mal verkleinert reporluziert. N.o. Nervus opticus. T3.g. Blutgefäsice.

Der grö̈sstr Kanal ist der Canalis opticus, wolcher fast in der Mitte des Bechers liegt. Seine Form ist rundlich, aber der Rand ist ganz höckerig, wenngleich nan darin kein، isolierten Knorpolinseln sieht, wis es beim vorigen Stadium der Fall ist.

Der Kanal besitzt durchschnittlich einen Durchmesser voln $0,4 \mathrm{~mm}(16 \mathrm{~mm})$, der dorsoventrale Durchmesser ist etwas klein. Er dient für den Durchtritt des S'chnerven und zweier anderer Blutgefüsse. Vor dem Eintritt zum Opticuskanal läuft der Opticus schrägr von temporodorsal nach nasorentral hin, einen Winkel ron etwa $60^{\circ}$ zwischen sich und der äusseren Basisfläche des Bechers bildend. Damn tritt er in den Kanal, ungefähr in der Mitte der hinteren Ausmündung ressclben ein. Im vorderen Ausmündungsteil kommt er zum nasalen Drittel und tritt nach einer leichten Biegung (Fin. 33, 35) in die Netzhaut ein. Man findet noch zwei Blutgefässe; das eine läuft an der Ventralscite les Opticus zu demselben parallel, das andere an der Temporalscite von unten nach oben damit kreuzend hin. Genau genommen ist die Lage dos Opticuskanal jedoch nicht in der Mitte des Becherbodens, wie die folgenden Masse es klar darstellen :

Dic Lage der hinteren Ausmündung des Canalis opticus selerae:

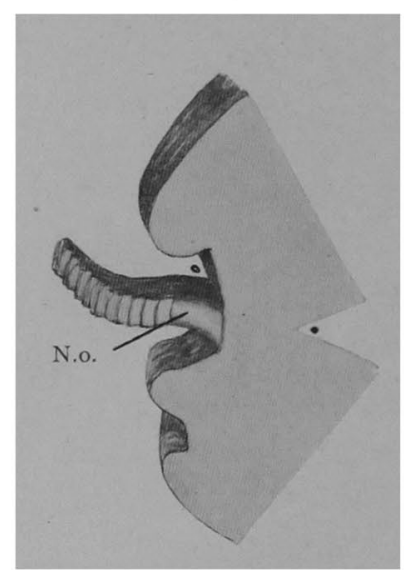

Fì. : :;:

Topographische In:icht des Nodells des Xervus opticus. N.o. Nervus opticus. 
a) Die Distanz zwischen dem Temporalrand des Canalis und dem Distalrand (les Knorpelbechers ca. 1,6 mm (64 mm).

b) Die Distanz zwischen den Nasalrand des Canalis und dem Distalrand des Knorpelbechers (a. $1,9 \mathrm{~mm}(76 \mathrm{~mm})$.

c) Die Distanz zwischen dem Ventralrand des Canalis und dem Distalrand des Knorpelbechers ca. $1,5 \mathrm{~mm}(60 \mathrm{~mm})$.

d) Die Jistanz zwischen dem Dorsalrand (les Canalis und dem I)istalıand des Knorpelbechers ca. $1,3 \mathrm{~mm}(52 \mathrm{~mm})$.

Die Lage der vorderen Ausmündung des Canalis opticus sclerae :

a) Die Distanz zwischen dem Temporalrand des Canalis und dem Distalrand des Knorpelbecher's ca. 1,4 $\mathrm{mm}(56 \mathrm{~mm})$.

b) Die Distanz zwischen dem Nasalıand des Canalis und dom Distalrand , les Knorpelbechers (a. 1,7 $\mathrm{mm}(68 \mathrm{~mm})$.

c) Die Distanz zwischen dem Ventralland des Canalis und dem Distalrand des Knorpelbechers ca. $1,3 \mathrm{~mm}(52 \mathrm{~mm})$.

d) Die Distanz zwischen dem Dorsalrand des Canalis und dem Distalrand des Knorpelbechers ca. $1,1 \mathrm{~mm}$ (44 mm).

Die zwei (icfässkanäle befinden sich nebeneinander auf der dorsalen Fläche des Bechers, von Canalis opticus ca. 0,2 mm, ein anderer Gefäisskanal auf der ventralen Fläche, davon ca. 0,6 mm und noch ein anderer (refïsskanal auf der rentronasalen Fläche, davon ca. 0,4 mm entfernt.

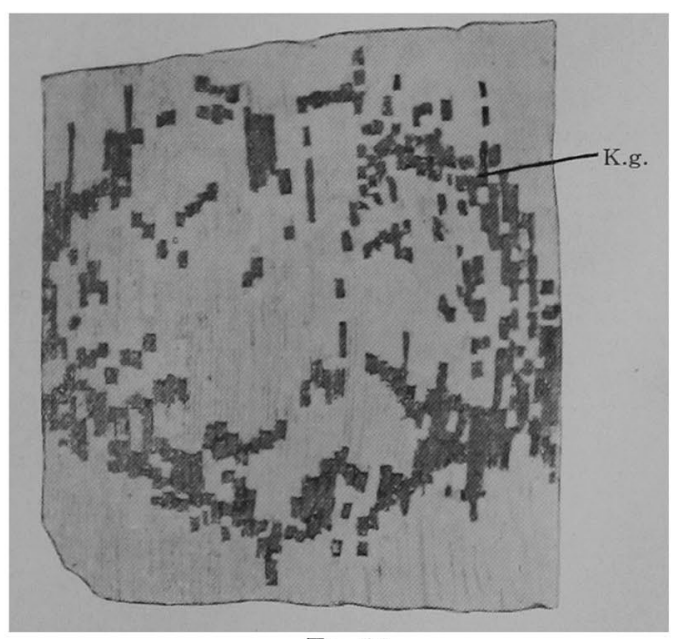

Fg. :3t.

Laterale Ansicht des Modells des rechten Auges. Stadium 10.40 mal vergrössert modelliert und auf 20 mal verkleinert reproduziert. K.g. Kornealgefässe.
In Bezur auf die Kornoalgefässe kamn man bei einem 40 fach vergrössert hergestellten Wachsmodell (Fig. 34) folgendes ersehen.

Die Kornealgefässse bilden ziemlich dichte Netzwerke im peripherischen Teil der Kornea, besonders in temporalen Teil. Aus diessen Kapillarschlingren gehen so viele Kapillarfortsätze aus, die teils einige kleine Kapillarringe bilken, toils frei endigen. Einer von diesen Kapillarfortsätzen ist schon zur Mitte der Kornea rorgedrungens. In diesem 
Stadium kann man nicht mehr cinen doppelt gefornten Randschlingenring wie beim vorigen Stadium erkennen. Dagregen sind dis Gofässnetze

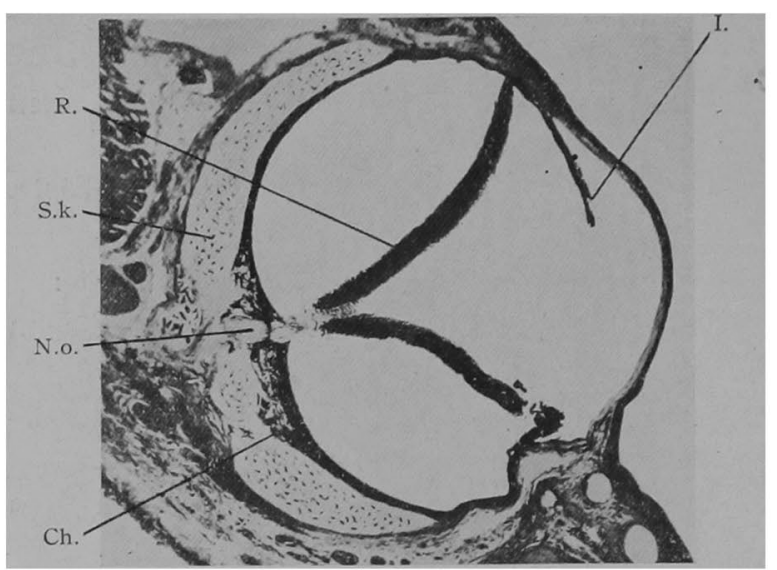

Fg. : :. . .

Frontaler Durcherhnitt des rechten Auges. (Ntadium 10, Virer. (al. 20 mal). S.k. Skleralknorpel. X.o. Xerwo esticus. ('h. Chorioideal. Ii. Retina. I. Iris.

achı" parallel zu seiner Oberfläche (Fig. 35). Hier unr da sicht num ganz spörliche pigmenticrto Knorpolyellen.

Bezüglich der Linse findet man keine besonl’’’n V'eränılerungen im Vergleiche mit dem rorigen Stadium. Was die Retina anbetrifft, so sind beille Kömerschichten viel deutlicher differnziert; man kann sogar eine (ranglicnzcllonschicht wanz klar unterscheiden. Die Choriodea hat bedeutend an Dicke zugenommen, besonder's in der Nähe des Becherbodens, worin einige Bluteföise enthalten sind. Die Iris ist stark pigmentiert und sieht wie die Chorioida schwärzlich aus.

Die Kornea (Fim. 36, 37) besteht nun aus 3-4 schichtigen niedrigen Epithelzellen und man kamn darin keine Pigmentkörner vorfinden. Die Substantia propria ist ziemlich gut entwickelt und hat nun die toppelte Dicke der Epithelschicht.

In ler Rạndzone der Sulsstantia sieht man ventral, wo sie im rorigen Stadium an clicksten waren, beleutend schwächer auscrobildet.

Mikroskopisch: In dor Mittelschicht des Knorpels ist die (irundsubstanz reichlicher zu finden, worin dis Knorpolzellen in vorshiedenen Formen liogen. Dic Zollen sind auch in vorshiedenen Richtungen angeorluet, doch in der Nïhe ler Oberfläche des Knorpels noiren ir sich nit ihrer Iä̈ngs-

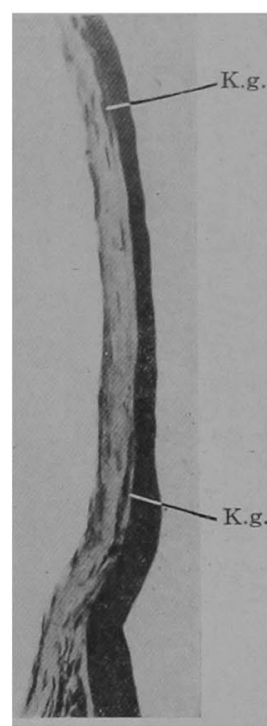

Fg. 36.

Frontaler Durchschnitt der Kornea des rechiteri Auges. (Natalium 10), Veror. ("a. 95 mal). K.g. Kornealgefïse. 


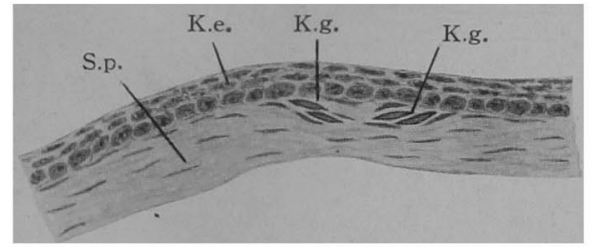

Fin. 37 .

Frontaler Durehschnitt der Korne:ar des rechten Auges. (Ntaclium 10, Vergr. ca. $200 \mathrm{mal})$. K.g. Kornealgefässce. K.e. Kornealepithel. S.p. Substantia propria. spärliche Pigmentkörner und man kam auch in der schicht nach der. Lage zwoi Arten von Kapillaren unterscheiden. Bei der ersten Art erkemnt man die clicht subepithelial durchzishonden Kapillaren, während boi der zweiten dic letzteren in obreffächlichen Drittel der Substantia vorkommen.

\section{Vergleichung und Zusammenfassung.}

1. a) Augenblise und Augenbecher.

Bei der 8,5 mm langen Larve von Menalobatrachus kommt gerade die primäre Aurenblase zum Vorschein. Bezüglich der Art und Weise der Bildung dersolben sieht man ähnlich wie bei den anderen Imphibien nichts beronderess als dass sie durch die dusbuchtung der ت̈usseren Torderhirnwand entsteht; dabei verbindet sie sich auf breiter Basis mit der letzteren. Yanche Zellen der Blasenwand enthalten spärliche Pigmentkörner. Die Augenblasentvand hat beinahe gleicho Dicke an allen Stellen; dieser Befund stimmt nicht mit der Angabe von Alexander (1) bei Siredon pisciformis überein, wonach sic sine viel dümmere Dorsalwant als dic ventrale besitzt. Die Blasenlöhle kommuniziert durch die weite Augenstiellöhle mit dem Vorderhirnventrikel.

Bei der $11 \mathrm{~mm}$ langen Lanre ist dic Augenblase schon zum Augenbecher umgestaltet und dessen äussere Wand bedeutend rordickt und leicht nach innen eingezogen, während die inmere äusserst dümn ist. Die Grundschicht des Ektoderms, die auf der äusseren Wand des Bechers liegt, ist ziemlich deutlich rerdickt und deutet die Linsenplatte an; die Dockschicht beteiligt sich dabei durchaus nicht an der Linsenbildung.

b) Augensticl und Sehnerv.

Die breite Basis der primäron Augenblasce ist bedeutond kleiner lxi $11 \mathrm{~mm}$ langer Larve und stellt clen Augensticl dar, der sich immer mehr verschmälert und innen einen ganz schmalen Sticlkanal enthält. Erst bei $23,5 \mathrm{~mm}$ langer Larve findet man dir Fascrzüge des Selnnerven in der Stielwand, wenngleich der Stiel noch ein offenes Stirlkanälchen enthı̈̈lt. Bei $30 \mathrm{~mm}$ langer Larve findet man zum ersten Mal cincu vollkommen ausarebildeten, soliden Nerrus opticus. Bei $80 \mathrm{~mm}$ und 179) $\mathrm{mm}$ langen Exemplaren zeigt der Opticus eine Biewung, beror er in dis Netzhaut eintritt. 
c) Kornea.

Bei den jüngeren Larven besteht das Epithel der Kornea aus zwoischichtigen Plattenzellen, die Substantia propria ist kaum sichtbar. Bei $30 \mathrm{~mm}$ langer Larre besteht das Epithel aus einer äusseren kubischen und einer inneren platten Zelle. Bei $80 \mathrm{~mm}$ langem Exemplar findet man erst ein aus 3-4 Zellenschichten bestehendes Hornhautepithel. Die Substantia propria ist ausserordentlich gut entwickelt, hat ungrefähr gleiche Dicke wie die Epithelschicht, und man sieht darin spärliche Pigmentkörner. In der oberflächlichsten Schicht der Propria von Amphibien fand auch Lauber (12) die Einlagrerung von Pigment. In der Ranlzone der Korna findet man bereits aufgetrotene Blutgefässe. Auf drer Innenfläche der Kornea kann man das Endothel deutlich erkemnen. Bei $179 \mathrm{~mm}$ langem Exemplar besteht die Epithelschicht aus 3-4 nierlrigen Zellenschichten. Die Propria ist so gut entwickelt, dass sio nun dio doppelte Dicke des Epithels beträgt, und die langen Plattenzcllen sind in 4-5 Reihen angeordnet. Die Komealgefässe sind sohr stark entwickelt, so dass ein Fortsatz schon zur Mitte der Kornea vorgedrungen ist.

d) Linse.

Die Linsenplatte, die erst bei $11 \mathrm{~mm}$ langer Larre aufwrtreten ist, wandelt sich bei $19 \mathrm{~mm}$ langer Larve zur Linsenblase um. Bei 20,5 mm langer Larve sieht man die etwa ellipsoidisch umgefornte Linse, die bei $26,5 \mathrm{~mm}$ langer Larve wierler eine rundliche Gestalt bekommt. Diese vorübergehenrle Ungestaltung der Linse fand Rabl (6) schon bei Siredon pisciformis. Bei $30 \mathrm{~mm}$ langer Lare ist der Linsenraum mit Linsenfasern vollkommen ausgefüllt. Bei $80 \mathrm{~mm}$ langem Exemplar liegen die Kerne der Linsenfasern im hinteren Teil derselben, fast in der Nähe des Äquators beisammen und bilden eine Kernzone.

e) Pigmentschicht.

Bei $11 \mathrm{~mm}$ langer Larre besteht das Pigmentblatt noch aus 1-2 schichtigen pigmentfreien kubischen Zellen. Bei $19 \mathrm{~mm}$ langer Larre sieht man erst das aus einschichtigen Plattenzellen bestehende Pigmentblatt, welches hie und da mit feinen Pignnentkörnern versehen ist. $B(\cdot i$ 26,5 mm langer Larre nimmt der Pigmentgehalt so stark zu, dass das Blatt braun aussieht. Bei $30 \mathrm{~mm}$ langer Larve bekommt es eine schwärzlichbraune Farbe durch starke Pigmentation. Bei $80 \mathrm{~mm}$ langem Exemplar ist die Ablagerung der Pigmentkömer bemerkenswert, indem die letzteren grösstenteils in der Nähe der Lussenfläche des Blattes angehäuft sind. Bei $179 \mathrm{~mm}$ langem Exemplar ist es bedeutend stärker pimnontiert und sieht diffus schwärzlich aus.

f) Chorioidea. 
Boi 2:3,5 $\mathrm{mm}$ langer Larve findet man erst das Chorioidealblatt, welches an ler Aussenscite des Pigmentblattes als eine pigmenthaltige Plattenzellenschicht auftritt. Bei $30 \mathrm{~mm}$ langer Larve ist es gut entwickelt. Bei $80 \mathrm{~mm}$ langem Exemplar kann man in der ziemlich verdickten Chorioidea cingelagerte Blutgefässe vorfinden.

g) Rotina.

Boi $11 \mathrm{~mm}$ langer Larve zieht die laterale Wand der Augenblase nach innen ein und bildet crst ein aus mehreren Zellenschichten bestehendes Retinalblatt. Bei 23,5 mm langer Lerve kann man erst kaum 3 Zellenschichten unterscheiden, bei $26,5 \mathrm{~mm}$ langer Larve ausserdem deutlich noch zwei retikuläre Schichten.

In der Retina des Anfangsstadiums von Amphibien sah Rabl (21) eine schwache Pigmentierung; bei Megalobatrachus dagegen kamn man keine Pigmentkörner konstatier'n. Bei $30 \mathrm{~mm}$ langer Larve nimmt die Retina bedeutend an Dicke zu, und man kann eine gut entwickelte innere retikuläre Schicht und zum erstenmal die Sehzellenschicht erkennen. Bei $80 \mathrm{~mm}$ langem Exemplar ist die Retina fast vollkommen differenziert und trägt deutlich cine 2-3 schichtige äussere Körner-, cine 3-4 schichtige imnero Körner-, eine 1-2 schichtige Nervonzellen-, aine Sehzellen- und eine Nervenfaserschicht. B×i $179 \mathrm{~mm}$ langem Exemplar sieht man ausser den obigen Schichten noch eino aus einschichtigen Zellen bestehende Ganglienzellenschicht.

h) Iris.

Bri $11 \mathrm{~mm}$ langer Larve ist der Augenbecher noch ganz seicht und der Pupillarrand nur schwach angedeutet. Bei $19 \mathrm{~mm}$ langer Larve erkennt man deutlich den Pupillarrand, wo aber noch keine Pigmentkörner vorhanden sind. Bei $23,5 \mathrm{~mm}$ langer Larve erst kommt eine schwache Pigmentierung in Tris vor. Bei $30 \mathrm{~mm}$ langer Larve berührt der Irisrand sich mit der Linse. $B$ (i $80 \mathrm{~mm}$ langem Exemplar findet man dic Verteilung der Blutgefässe im Randteil der Iris.

\section{Das Vorkommen des Skleralknorpels.}

Die frïhzeitige Knorpelbildung in der Sklera von Amphibien wurde bis jetzt ron Stadtmüller (23), Okajima (18), Tsuzaki (18, 25) und Yano (28) u.a. verfolgt

Stadtmüller hat geäussert, dass bei Salamandra maculosa (rerschiedene Stadien), Salamandra atra (28 $\mathrm{mm}$ lange Larve), Triton spec. (31 mm lange Larve), Salamandra perspicillata, D) 
Amblystoma (34 $\mathrm{mm}$ lange Larve) der frühzeitige Skleralknorpel immer ringförmig auftritt.

Okajima und Tsuzaki (18) ehenfalls betonen, dass bei den Laren von Diemictylus pyrrhogaster, Hynobius nebulosus und Onychorlactylus japonicus der Skleralknorpel zuerst in ringförmiger Anordung zum Vorschein kommt.

Tsuzaki (25) beobachtet bei $1+\mathrm{mm}$ langer Larve von Dienictylus pyrrhogaster ein Knorpelstïck, welches an der Ventralwand des Augenhintergrundes aufgetreten ist.

Nach Yano (28) kommt bei jungen Tieren von Hyla, Rhacophorus, Polypedates, Rana und Bufo der Skleralknorpel immer stückwoise im temporoventralen Abechnitt des Augenhintergrundes vor. Er sagt dabei, dass er in der Nähe orler entsprechend der Insertions\%one der Augenmuskeln einige zorstreute neue Knorpelelemente rorgefunden habe. Und über die Verhältnisse zwisch'n den Augenmuskehn und dem Skkralknorfel sinl Gegenbaur (4) und Yano (28) dex gleichen Meinung, so rass beim gewaltigen Zug der Augenmuskehn der Bulbus einen pasconten Wirlorstand darbieten müsste.

Bezüglich der Reihenfolge des Erscheinens von Knorp@stücken sah Yano (28) bui Bufo, lass das Stück zuerst im temporalen Abschnitt des Augenhintergrundes und dann an dorsalen und nasalen auftritt.

Bei mir tritt der Skleralknorpel zuerst $h_{x i}$ der Jarve von $30 \mathrm{~mm}$ Gesamtlänge stückweise an der temporalen, dorsalen und nasalen Wand des Augenhinterereundes auf einmal auf und an der ventralen findet man kein Knorpelstück. Dieser Befund weicht von den Angaben nach Tsuzaki und Yano ab.

Auf Schnitten findet man ohne Ausnahme an der Aussenfläche aller Knorprelstücke die ansetzenden Augenmuskelfasern. Über das Vorhandensein des innigen Verhältnisses zwischen den Augenmuskeln und Stellen, wo das Knorpelstück zuerst auftritt, bin ich auch der glcichen Mnsicht wie Gegenbaur und Yano.

Alle diese frühen Knorpel enthalten keine Pigmentk̈̈rncr. Bei 80 mm langen Exomplar ist der Knorpelbecher schon ziemlich gut ausgebildet und ungefähn in der Mitte des Becherbodens findet sich ein unregelmässigr rundlicher Canalis opticus; ansserlem findet man einen kleinen Gefässkanal an der dorsalen und einen anderen an der ventralen Wand. Der Knorpel enthält jetzt geringe Pigmentkörner. Bei $179 \mathrm{~mm}$ langem Exemplar ist der Knorpelbecher sohr gut entwickelt. Fast in der Nitte des Bocherbordens stoht ein gerüumiger Canalis opticus, dessen Rand höckerign ist, aber keine isolierte Knorpelinsel trägt, die Yano (27) 
beim erwachsenen Tiere von Megalobatrachus beobachtet hatte. Ausser dem Opticuskanal sieht man zwei Gefässkanäle an der Dorsal-, einen an der Ventral- und noch einen an der Nasalwand des Bechers.

In Bezug auf dis Lage und Zahl der Gefässkanäle scheint es mir, dass es sich je nach den Starlien sehr verschieden verhält. Der Knorpel in diesem. Stadium enthält ganz greringe Pigmentkörner.

\section{Frühzeitige Hornhautvaskularisation.}

Rejsck (20) beschrieb zuerst die Hornhautvaskularisation bei Urodelen, worauf dann viele Untersuchungen folgten, von denen die Arbeiten von Lauber (13), Ito (7) und Tsuzaki (21) besonders hervorzuheben sind.

Ito untersuchte beim erwachsenen Megalobatrachus die Verbreitung und Lagerung der Hornhautgefässe eingehend und fand, dass die Gefässe in der Hornhaut durch die komplizierten Anastomosen arkadenförmig ausgebreitet sind. Nach ihm liegen sie in der Schicht zwischen dem oberflüchlichen Drittel und den tiefen /swei Dritteln der Substantia propria.

Tsuzaki (24) sah beim Dionictylus, dass die ventralen zwei Drittel der Hornhaut von Blutgefässen versorgt werlen, indem sie von ventral vorn nach oben dorsal in den arkadenförmigen Anastomosen verlaufen.

Was die Lage der Gefässce betrifft, so stimmt soine Angabe mit dersolben von Ito (7) vollkommen überein.

In meinem Falle findet man zuerst bei $80 \mathrm{~mm}$ langem Exemplar eine frühzeitige Vaskularisierung in der Randzone der Hornhaut, während keine Gefässe im mittleren Teil derselben vorkommen. Die vom Randteile eingedrungenen Gefässe bilden zwei Gofässringe, einen äusseren und einen inneren, die beide an der Temporalseite auf kurze Strecke unterbrochen sind. Aus dem inneren Gefässring ziehen einige Gefässfortsätze von vorn oben nach hinten unten hin. Aus obigen Befunden scheint es mir höchst wahrscheinlich, dass die Gefässfortsät\%o zuerst nach dem Hornhautzentrum zu eintreten und dam sich miteinander verbinden, um endlich beim ausgrwachsenen ein in ganzer Hornhaut aushritendes umfangreiches Netz zu bilden, wie es schon von früheren Autoren swesehen wurde.

Bezüglich der Tiefe der Lage von Gefässen stimmt es bei dicsem Tiere mit ler Angabe ron Ito und Tsuzaki überein. Bei 179 mm langem Exomplar ist das Randschlingennetz der Gefässe äussrst kompliziert geformt und man kann nicht mehr die Gefässsinge erkennen. Viele Gefässfortsätze 
streben von allen Seiten der Hornhautrand nach dem Hornhautzentrum zu und bilden ein kompliziertes Randschlingemetz. Einer von diesen Fortsätzen erreicht schon die Mitte der Hornhaut. Hier verhält sich die Lagn der Gefässe etwas anders als beim vorigen Exemplare, so dass man hier dic dicht subepithelialen Gefässe anstatt der ticfen Kapillaren findet; auf Schnitten beobachtet man ebenso zwë Arten von Blutgefässen, von denen die eine dicht subepithelial, dir andere im oberflächlichen Drittel der Propria sich befindet, und keine Gefässe in den tiefen zwei Drittclı erkenmbar sind. Dieser Befund stimmt mit der Angabe ron Lauber (13) vollkommen überein.

Was die Lagerung der Gefässe anbelangt, so glaube ich, dass sie sich auch je nach den Individuen und den Entwicklungsstadien sohr verschiedon verhält.

\section{Ergebnisse.}

Die Hauptresultate der rorliegenden Untersuchungen lassen sich folgendermassen zusammenfassen.

1. Die Dicke aller Wandteile der prinären Augenblase ist ungefähr gleich. Die Zellen Acr Augensticlwand heginnen sich bei $23,5 \mathrm{~mm}$ langer Larve teils zu Nervenfasern umzuwandeln, und bei $30 \mathrm{~mm}$ langer Larve ist der Opticus vollkommen ausgebildet. Der letztere erfährt einc Biegung, bevor er in die Net\%haut eintritt.

Die Linsenplatte entsteht bei $11 \mathrm{~mm}$ langer Larve durch die Verdickung der Grundschicht (les Ektoderms. Die kuglige Linsenblase ist schon boi $19 \mathrm{~mm}$ langer Larve völlig geformt und erweist sich b(i 20.5 $\mathrm{mm}$ langer Larve vorübergehend als ellipsoidisch, um bei $26,5 \mathrm{~mm}$ langer Larve wieder eine rundliche Form zu bekommen. Der Linsenblasenraum wird bei $30 \mathrm{~mm}$ langer Larve mit den Linsenfasern vollkommen ausgefüllt.

Das Korncalepithel wird zuerst bei $80 \mathrm{~mm}$ langem Exomplar mehrschichtig, wobei die Vaskularisierung der Kormea bereits begonnen hat. Die Chorioidea wird zuerst bei $23,5 \mathrm{~mm}$ langer Larve gebildet. Dic Iris ist erst bei $80 \mathrm{~mm}$ langem Exemplar geformt. Das Pigmentblatt weist erst bei $19 \mathrm{~mm}$ langer Larve die Pigmentation auf. Die einzelnen Retinalschichten sind bei $26,5 \mathrm{~mm}$ langer Larve klar unterseheidbar.

2. Der Skliralknorpel kommt stïckweise erst an der temporalen, dorsalen und nasalen Wand des Augenhintergrundes zum Vorschein; die Stellen, wo er vorkommt, entsprechen der Insertionszone der Augrenmuskch. Er enthält in früheren Stadien kcin Pigment. 
3. Die Vaskularisation der Hornhaut entsteht zuerst im Randteil drselben. Dam breiten sich die Blutgefässfortsätze nach der Mitte der Hornhaut zu aus. Dic Gefässfortsätze verbinden sich miteinander und bilden ein arkadenförmiges Gefässnetz. Die Tiefe der Lage von Gefässen in der Substantia propria ist je nach den Stadinn ganz verschieden, so dass man bei einem Tiere lie tief gelegenen und beim anderen Tiere (lafür die dicht subepithelial golegenen Kapillaren vorfindet.

Zum Schluss möchte ich Herrn Prof. K. Okajima für seine freundliche Anregung und Unterstützung bei dieser Arbcit meinen herzlichsten Dank aussprechen.

\section{Literatur.}

1. Alexander, J., Zur Entwicklungsgewchichte des Wirbelticrauges. Mnat. Anz. Bd. j). 1918.

2. Barkau, J., Beiträge zur Entwicklungsgeschichțe des Auges der Batrachier. Sitz13.r. Akad. Wiss. Wien, math.-nat. Kl. I.IV. Lbt. 1. 1866.

:3. Götte, А., Kurze Mitteilungen aus der Entwicklungsgeschichte der Unke. Arch. f. mikr. Inat. Bd. IX. 187?.

4. Gegenbaur, C., Vergleichende Inatomie der Wirbeltiere. Bd. 1. 1898.

5. Hertwig, O., I, Hrbuch der Entwicklungsgeschichte des Menschen und der Wirbeltiere. 10. Iufl. 1915.

6. - Handbuch der vergl. u. experin. Entwicklungstehre. Bd. 2. II. Teil. 1909.

7. Ito, II., Die (iefïsse und die Pigmentzellen der Hornhant bei MIegalobatrachus japonicus. Ḱyoto-Isen-Koyukaizasshi. Nr. 51. 1909.

s. Joseph, H., Finige Bemerkungen zu F. Maurer's Mbhandlung: „, Blutgefïser im Epithel." Arch. f. mikr. Inat. Bd. 5’. 1898.

9. Kunitomo, K., Über Blutwefässe in cler Epidermis von Megalobatrachus japonicus. Mitteil. 1. med. (iesellsch. zu Tokyo. Bd. 17. 1903.

10. Kingsley, J. S., Comparative Anatomy of Vertebrates. ind Edit. 1912.

11. Leydig, F., Vaskularisiertes Epithel. Arch. f. mikrosk. Anat. Bd. 5:. 1898.

12. La uber, H., Beiträge zur Inatomie des vorderen lugenaloschnittes der Wirbeltiere. Inat. Ilefte. Heft I.IX. 1902.

13. - Inatomische Untersuchung des Auges von Cryltobranchus japonicus. Anat. Hefte. Heft. I.XIV. 190\%.

14. de Lange, D., Mitteilungen zur Fntwicklungsgeschichte des japani-chen Riesensalamanders (Meg. maximus S.hlegel). Inat. Anz. Bd. 43. 1913.

15. Murata, MI., Über die Verbreitungsweise der Blutkapillaren in den inneren Oramen von Cryptobranchus japonicus. Mitteil. d. med. Fakl. d. Kais. Jap. Univ. zu Tokyo. Bd. 9. Heft. ?. 1909.

16. Maurer, F., Blutgefässe im Epithel. Morph. Jahrb. Bd. 25. 1897. 
17. Okajima, K., Untersuchung über die Sinnesorgane von Onychorlactylus. Zeitschr. f. wj.s. Zool. Bd. 94. 1909.

18. Okajima, K., und Tsuzaki, T., Beiträge zur Morphologie des Skleralknorpels bei den Urodelen. Zeitschr. f. Anat. und Intw. Bd. 60. 1921.

19. Osaki, D., Zur Frage des Vorkommens les Şkleralknorpels bei Diemictylus. Folia anat. japon. Bd. 3. 1925.

20. Rejsek, J., L'histologie de l'oeil de cryptobranchus japonicus. Bibliographic anatomique. 1897.

21. Rabl, C., Üher den Bau und die Entwicklung der Linse. Zeitschr. f. wiss. Zool. Bd. IXXIII. 1898.

22. Rüdinger, N., Über die Billung der primären und sekundären Augenblasen bei Triton alpestris. Sit\%. Ber. math.-physik. Kl. Akad. Wiss. Bd. XIX. 1889.

2:). Stadtmüller, F., Fin Beitrag zur Kenntnis des Vorkommens und der Bedeutung hyalinknorpeliger Elemente in der Sklera der Urodelen. Anat. Hefte. H. $15 t$ (Bd. 51. H. 2.) 1914 .

24. Tsuzaki, T., Zur MIorphologie des Skleralknorjels von Diemictylus pyrrhogaster. Folia anat. japon. Bd. I. 192:.

25. Tsuzaki, T., I)ie Hornhautvaskularisation bei Diemictylus pyrrhogaster. Folia anat. japon. Bd. II. 19:4.

26. Wiedersheim, R., Vergleichende Anatomie der Wirbeltiere. 7. Aufl. 1909.

27. Yano, K., Zur Morphologie des Skleralknorpels und -knochens bei den Trodelen. Folia anat. japon. Pd. 1V. 1926.

2s. Yano, K., Die Fntwicklung und Morphologie des. skleralknorpels bei den Anuren. Folia anat. japon. Bd. V. 1927. 